\title{
The impact of "early" versus "late" initiation of renal replacement therapy in critical care patients with acute kidney injury: a systematic review and evidence synthesis
}

\author{
Benjamin T. Wierstra', Sameer Kadri², Soha Alomar², Ximena Burbano², Glen W. Barrisford ${ }^{2}$ \\ and Raymond L. C. Kao $2,3^{*}$
}

\begin{abstract}
Background: The optimal timing of initiating renal replacement therapy (RRT) in critical illness complicated by acute kidney injury (AKI) is not clearly established. Trials completed on this topic have been marked by contradictory findings as well as quality and heterogeneity issues. Our goal was to perform a synthesis of the evidence regarding the impact of "early" versus "late" RRT in critically ill patients with AKl, focusing on the highest-quality research on this topic.
\end{abstract}

Methods: A literature search using the PubMed and Embase databases was completed to identify studies involving critically ill adult patients with AKI who received hemodialysis according to "early" versus "late"/"standard" criteria. The highest-quality studies were selected for meta-analysis. The primary outcome of interest was mortality at 1 month (composite of 28- and 30-day mortality). Secondary outcomes evaluated included intensive care unit (ICU) and hospital length of stay (LOS).

Results: Thirty-six studies (seven randomized controlled trials, ten prospective cohorts, and nineteen retrospective cohorts) were identified for detailed evaluation. Nine studies involving 1042 patients were considered to be of high quality and were included for quantitative analysis. No survival advantage was found with "early" RRT among high-quality studies with an OR of 0.665 ( $95 \% \mathrm{Cl} 0.384-1.153, p=0.146$ ). Subgroup analysis by reason for ICU admission (surgical/medical) or definition of "early" (time/biochemical) showed no evidence of survival advantage. No significant differences were observed in ICU or hospital LOS among high-quality studies.

Conclusions: Our conclusion based on this evidence synthesis is that "early" initiation of RRT in critical illness complicated by AKI does not improve patient survival or confer reductions in ICU or hospital LOS.

Keywords: Meta-analysis, Intensive care units (ICUs), Acute kidney injury (AKI), Renal replacement therapy (RRT), Early, Late

\section{Background}

Acute kidney injury (AKI) is a medical complication associated with significant morbidity and mortality in critically ill patients [1-3]. AKI is common in critical illness, and severe AKI is associated with up to $60 \%$ hospital mortality [4]. Renal replacement therapy (RRT)

\footnotetext{
* Correspondence: rkao3@uwo.ca

${ }^{2}$ Harvard School of Public Health, Harvard University, Boston, MA, USA ${ }^{3}$ Division of Critical Care Medicine, Department of Medicine, Western University, 800 Commissioner's Road East, London, ON N6A 5W9, Canada

Full list of author information is available at the end of the article
}

within the intensive care unit (ICU) is conducted as either intermittent hemodialysis or continuous renal replacement therapy (CRRT). Traditional indications for RRT require the development of overt clinical manifestations of renal insufficiency, such as acidosis, electrolyte disturbances (most notably hyperkalemia), uremic complications (encephalopathy or pericarditis), and volume overload unresponsive to aggressive medical management. In spite of research and increasing clinical experience with dialysis, the optimal time to initiate RRT in the course of critical illness complicated by AKI is unclear. 
The notion of "early" RRT is to initiate dialysis therapy before nitrogenous and other metabolic products accumulate to the degree where they become relatively resistant to therapy $[5,6]$. Despite the intuitive rationale for "early" RRT, there is limited evidence to guide clinicians on the optimal time to initiate RRT in critical illness. Neither standard clinical parameters nor research into novel clinical biomarkers has emerged to clearly define an ideal time or clinical picture where the initiation of RRT optimizes patient outcomes. Earlier initiation of RRT must be balanced with potential patient harm associated with RRTs. Protocolled use of hemofiltration for $96 \mathrm{~h}$ in patients with septic shock admitted to an ICU regardless of their renal function suggests that "early" RRT can be associated with negative patient outcomes [7]. As a result, research into "early" RRT includes multiple definitions of early that reflect a potpourri of time factors, biochemical markers, and clinical parameters in an attempt to balance the risks of initiating RRT with the benefits expected from supporting renal function during critical illness.

The authors of two earlier meta-analyses pooled available data on this topic to suggest that "early" RRT improves survival in critical illness. Seabra et al. [8] identified 23 studies (5 randomized controlled trials [RCTs]/ quasi-RCTs, 1 prospective study, and 17 retrospective cohort studies) and concluded that "early" initiation of RRT was associated with $28 \%$ mortality risk reduction (relative risk [RR] 0.72, $95 \%$ CI 0.64-0.82, $p<0.001$ ). Karvellas et al. [9] identified 15 studies ( 2 RCTs, 4 prospective studies, and 9 retrospective cohort studies) and reached similar conclusions, reporting a significant improvement in 28-day mortality with "early" RRT (OR $0.45,95 \%$ CI $0.28-0.72, p<0.001)$. However, the overall findings were not congruent with the subgroup analysis of randomized trials (RR 0.64, $95 \%$ CI $0.4-1.05, p=0.08$ ), where there was a signal that "early" RRT was not associated with a significant survival advantage. This has diminished clinical confidence in the conclusions reached by the earlier meta-analyses, and consequently "early" RRT in critical illness remains a controversial therapeutic intervention.

Since 2012, additional studies have been published that do not support the conclusions of the previous metaanalyses, and this has further diminished the confidence in the previous conclusions that suggested a survival benefit in critical illness associated with "early" RRT. We conducted a systematic review and evidence synthesis to investigate whether "early" versus "late" initiation of RRT in critically ill patients with AKI improves patient survival and selected secondary outcomes for potential signals to suggest that "early" RRT may reduce patient morbidity or enhance illness recovery. Our goal was to identify the highest-quality studies on this topic and use a pooled meta-analysis of these studies to inform our conclusions.

\section{Methods \\ Search strategy}

This study was conducted in accordance with the Preferred Reporting Items for Systematic Reviews and MetaAnalyses (PRISMA) guidelines [10] (see Additional file 1: Figure S1 for PRISMA checklist). Our null hypothesis was that "early" initiation of RRT does not improve patient survival in critical care patients with AKI. This systematic review was not registered, and a protocol does not exist. The PubMed and Embase databases were searched to identify published articles following four broad themes: AKI, RRT, time of initiation, and critical illness (see Additional file 2: Table S1 for search terms). SK is a National Institutes of Health $(\mathrm{NIH})$ physician and requested the NIH librarian to provide oversight for the search strategy. Our search was limited to Englishlanguage-only, full-text primary research publications (including abstracts with full text availability) reporting findings of clinical trials and observational studies (cohort and case-control design) published between January 1985 and November 2015. Studies before 1985 were not actively sought, owing to a low likelihood of relevance to modern RRTs and critical care practices.

\section{Study selection}

References were screened and excluded if they were small case reports or observational studies (fewer than 10 subjects), were not focused on critically ill adult patients, did not report mortality data, involved basic science data, or did not clearly distinguish between "early" and "late" groups. This task was divided among the authors. A second evaluation led by the senior author (RLCK) was conducted to evaluate study quality. Studies were designated as being of "high quality" or "low quality." Studies were assigned a "low-quality" rating if there was no illness severity assessment between cohorts or at the time of randomization $(n=8)$, significant differences $(p<0.05)$ between cohort groups $(n=7)$ at baseline, incomplete basic demographic data at baseline $(n=6)$ to exclude baseline differences, or a NewcastleOttawa Quality Assessment (NOQA) Scale [11] for cohort studies rating less than $7(n=6)$. The senior author (RLCK) was the arbiter in cases of disagreement. Only high-quality studies were included in quantitative metaanalysis of the primary and secondary outcomes.

\section{Primary and secondary outcomes}

The primary outcome of interest was mortality at 1 month (pooling outcomes for mortality at 28 or 30 days, depending on what was reported by the primary authors). In addition to mortality, we analyzed selected secondary 
Table 1 Trial Summary Table by Study Type $(n=36)$

\begin{tabular}{|c|c|c|c|c|c|c|c|c|c|c|c|c|c|c|}
\hline \multirow[t]{2}{*}{ Author, Year } & \multirow[t]{2}{*}{ Study Design } & \multirow[t]{2}{*}{ Country } & \multirow[t]{2}{*}{ Duration } & \multirow[t]{2}{*}{ Exclusion } & \multirow{2}{*}{$\begin{array}{l}\text { Patient } \\
\text { Population }\end{array}$} & \multicolumn{3}{|c|}{ Patients (n) } & \multirow{2}{*}{$\begin{array}{l}\text { Age } \\
\text { (mean) } \\
\text { yrs }\end{array}$} & \multirow{2}{*}{$\begin{array}{l}\text { Illness Severity } \\
\text { Score }\end{array}$} & \multirow{2}{*}{$\begin{array}{l}\text { Early RRT } \\
\text { Criteria }\end{array}$} & \multirow{2}{*}{$\begin{array}{l}\text { Late RRT } \\
\text { Criteria }\end{array}$} & \multirow{2}{*}{$\begin{array}{l}\text { Study } \\
\text { Quality }\end{array}$} & \multirow{2}{*}{$\begin{array}{l}\text { Primary } \\
\text { Outcome }\end{array}$} \\
\hline & & & & & & $\overline{\text { Total }}$ & $\begin{array}{l}\text { Early } \\
\text { RRT }\end{array}$ & $\begin{array}{l}\text { Late } \\
\text { RRT }\end{array}$ & & & & & & \\
\hline \multicolumn{15}{|c|}{ Randomized Trials } \\
\hline $\begin{array}{l}\text { Bouman, } \\
2002[12]\end{array}$ & $\begin{array}{l}\text { RCT, } \\
\text { two-center } \\
\text { study }\end{array}$ & Netherlands & $\begin{array}{l}\text { May } 1998 \text { - } \\
\text { Mar } 2000\end{array}$ & $\begin{array}{l}\text { Pre-existing } \\
\text { renal disease }\end{array}$ & Multisystem & 106 & 70 & 36 & $\begin{array}{l}\text { EHV: } 68 ; \\
\text { ELV: } 70 ; \\
\text { LLV: } 67\end{array}$ & $\begin{array}{l}\text { EHV: SOFA } 10.3 \text { - } \\
\text { APACHE2 }=23.5, \\
\text { ELV: SOFA } 10.1 \text { - } \\
\text { APACHE2 }=21.7 ; \\
\text { LLV: SOFA } 10.6 \text { - } \\
\text { APACHE2 }=23.6\end{array}$ & $\begin{array}{l}\text { TIME: Early } \\
<12 \mathrm{~h}(200 \mathrm{mll}) ; \\
\text { Early Low Vol } \\
<12 \mathrm{~h}(100- \\
150 \mathrm{ml})\end{array}$ & $\begin{array}{l}\text { TIME: Late } \\
>12 \mathrm{~h}\end{array}$ & $H I G H$ & $\begin{array}{l}28 \text { d mortality: } \\
\text { EHV: } 9 / 35(26 \%) \text { died, } \\
\text { ELV: } 11 / 35(31 \%) \text { died, } \\
\text { LLV: } 9 / 36(25 \%) \text { died; } \\
p=0.8\end{array}$ \\
\hline $\begin{array}{l}\text { Durmaz, } \\
2003[13]\end{array}$ & RCT & Turkey & $\begin{array}{l}\text { Sept } 1999 \text { - } \\
\text { Aug } 2001\end{array}$ & $\begin{array}{l}\text { Age }<18, \\
\text { chronic } \\
\text { dialysis }\end{array}$ & $\begin{array}{l}\text { Post Cardiac } \\
\text { Surgery }\end{array}$ & 44 & 21 & 23 & $\begin{array}{l}\text { Early } 58 ; \\
\text { Late } 54\end{array}$ & NR & $\begin{array}{l}\text { BIOCHEM: Cr } \\
\text { rise }>10 \% \text { from } \\
\text { pre-op level } \\
\text { within } 48 \text { hrs } \\
\text { of surgery }\end{array}$ & $\begin{array}{l}\text { Cr rise }>50 \% \\
\text { from pre-op level; } \\
\text { or Urine output } \\
<400 \mathrm{~m} / 24 \mathrm{hrs} \\
\text { with coexistent } \\
\mathrm{K}+/ \mathrm{H}+\text { unresponsive } \\
\text { to med mgmt }\end{array}$ & LOW & $\begin{array}{l}\text { Hospital mortality: } \\
\text { Early } 1 / 21(4.8 \%) \text { died, } \\
\text { Late } 7 / 23(30.4 \%) \text { died } \\
p=0.048 \text {; Favors Early }\end{array}$ \\
\hline $\begin{array}{l}\text { Sugahara, } \\
2004[14]\end{array}$ & RCT & Japan & $\begin{array}{l}\text { Jan } 1995- \\
\text { Dec } 1997\end{array}$ & $\begin{array}{l}\text { Pregnancy, Bili } \\
>5 \mathrm{mg} / \mathrm{dL} \text {, Mental } \\
\text { disorder, Cancer, } \\
\text { Early recovery of } \\
\text { urine output } \\
>30 \mathrm{~m} / \mathrm{kg} / \mathrm{hr} \\
\text { prior to RRT }\end{array}$ & $\begin{array}{l}\text { Post Cardiac } \\
\text { Surgery }\end{array}$ & 28 & 14 & 14 & $\begin{array}{l}\text { Early: } 65 ; \\
\text { Late: } 64\end{array}$ & $\begin{array}{l}\text { Early: APACHE2 }=19 ; \\
\text { Late: APACHE2 }=18\end{array}$ & $\begin{array}{l}\text { BIOCHEM: UOP } \\
<30 \mathrm{~m} / / \mathrm{hr} \times \\
\text { 3hrs OR UOP } \\
<750 \mathrm{~m} / / \text { day; } \\
\text { Mean time to } \\
\text { RRT start } \\
18 \text { d } \pm 0.9 \text { post op }\end{array}$ & $\begin{array}{l}\text { UOP }<20 \mathrm{ml} / \mathrm{hr} \times \\
2 \text { hrs } \mathrm{OR} \text { UOP } \\
<500 \mathrm{~m} / \mathrm{day} ; \\
\text { Mean time to RRT } \\
\text { start } 1.7 \mathrm{~d} \pm 0.8 \text { post op }\end{array}$ & HIGH & $\begin{array}{l}14 \mathrm{~d} \text { mortality: Early } 2 / 14 \\
\text { died (14\%), Late 12/14 } \\
\text { died (86\%); } p<0.01 \\
\text { Favors Early }\end{array}$ \\
\hline $\begin{array}{l}\text { Payen, } \\
2009[7]\end{array}$ & $\begin{array}{l}\text { RCT, } \\
\text { multicenter }\end{array}$ & France & $\begin{array}{l}\text { Jan } 1997- \\
\text { Jan } 2000\end{array}$ & $\begin{array}{l}\text { Age< } 18 \text {, chronic } \\
\text { dialysis, pregnant, } \\
\text { moribund state, prior } \\
\text { immunosuppressive } \\
\text { therapy }\end{array}$ & Multisystem & 76 & 37 & 39 & Early 58 Late 59 & $\begin{array}{l}\text { Early: SOFA 11.6- } \\
\text { SAPS2 54.3; } \\
\text { Late: SOFA 10.4- } \\
\text { SAPS2 } 52.4\end{array}$ & $\begin{array}{l}\text { TIME: } \\
\text { Protocolized } \\
\text { RRT } \times \text { 9 } 6 \text { hrs } \\
\text { w/ diagnosis of } \\
\text { 'sepsis'. Mean } \\
\text { time to initiation } \\
\text { of RRT not specified }\end{array}$ & $\begin{array}{l}\text { Control = No RRT } \\
\text { unless metabolic } \\
\text { renal failure \& classic } \\
\text { indications for RRT } \\
\text { present }\end{array}$ & $H I G H$ & $\begin{array}{l}\text { Early } 20 / 37(54 \%) \text { died, } \\
\text { Late } 17 / 37(44 \%) \text { died; } \\
p=0.49\end{array}$ \\
\hline $\begin{array}{l}\text { Jamale, } \\
2013[15]\end{array}$ & $\begin{array}{l}\mathrm{RCT} \text {, single } \\
\text { center }\end{array}$ & India & $\begin{array}{l}\text { April } 2010 \text { - } \\
\text { July } 2012\end{array}$ & $\begin{array}{l}\text { Required urgent } \\
\text { dialysis at time } \\
\text { of randomization }\end{array}$ & Multisystem & 208 & 102 & 106 & Early 43 Late 42 & $\begin{array}{l}\text { Early: SOFA 7.3; } \\
\text { Late: SOFA 8.2 }\end{array}$ & $\begin{array}{l}\text { BIOCHEM: Cr } \\
>618 \mu \mathrm{mol} / \mathrm{L}\end{array}$ & $\begin{array}{l}\text { Classic indications } \\
\text { for RRT, Symptomatic } \\
\text { uremia unresponsive } \\
\text { to med mgmt }\end{array}$ & HIGH & $\begin{array}{l}\text { Mortality: Early 21/102 } \\
\text { (20.5\%) died, } \\
\text { Late 13/106 (12\%); } \\
p=0.2\end{array}$ \\
\hline $\begin{array}{l}\text { Combes, } \\
2015[16]\end{array}$ & $\begin{array}{l}\text { RCT, } \\
\text { multicenter }\end{array}$ & USA & 2009-2012 & $\begin{array}{l}<18 \text {, Pregnant, } \\
\text { Chronic RRT, } \\
\text { Weight }>120 \mathrm{~kg} \text {, } \\
\text { SAPS II>90 } \\
\text { (i.e. moribund) }\end{array}$ & $\begin{array}{l}\text { Post Cardiac } \\
\text { Surgery }\end{array}$ & 224 & 112 & 112 & Early 61 Late 58 & $\begin{array}{l}\text { Early: SOFA } 11.5- \\
\text { SAPS2 }=54 ; \\
\text { Late: SOFA } 12.0- \\
\text { SAPS2 } 2=55.1\end{array}$ & $\begin{array}{l}\text { TIME: RRT initiated } \\
<24 \mathrm{hrs} \text { and continued } \\
\text { for min of } 48 \mathrm{hrs} \text {; Mean } \\
\text { time to randomization } 12 \mathrm{hrs}\end{array}$ & $\begin{array}{l}\text { Classic indications } \\
\text { for RRT, Lifethreatening } \\
\text { metabolic derangements } \\
\text { unresponsive to med mgmt }\end{array}$ & HIGH & $\begin{array}{l}\text { Mortality: Early 40/112 } \\
(36 \%) \text { died, Late 40/112 } \\
(36 \%) \text { died; } p=1.0\end{array}$ \\
\hline $\begin{array}{l}\text { Wald, } \\
2015[17]\end{array}$ & $\begin{array}{l}\text { RCT, } \\
\text { multicenter }\end{array}$ & Canada & $\begin{array}{l}\text { May } 2012 \text { - } \\
\text { Nov } 2013\end{array}$ & $\begin{array}{l}\text { Intoxication } \\
\text { requiring RRT, } \\
\text { Limited resuscitation } \\
\text { directives, RRT within } \\
\text { the previous } 2 \text { months, } \\
\text { RPGN, Obstructive } \\
\text { uropathy, }>48 \mathrm{hrs} \text { to } \\
\text { doubling time of } \mathrm{Cr}\end{array}$ & Multisystem & 100 & 48 & 52 & Early 62 Late 64 & $\begin{array}{l}\text { Early: SOFA } 13.3 \\
\text { Late: SOFA } 12.8\end{array}$ & $\begin{array}{l}\text { TIME: Time from } \\
\text { randomization } \\
<12 \text { h; Mean time } \\
\text { to RRT = 9.7hrs }\end{array}$ & $\begin{array}{l}\text { Intensivist judgement } \\
\text { regarding hyperkalemia, } \\
\text { volume overload, acidemia } \\
\text { refractory to medical therapy, } \\
\text { Uremic symptoms Mean } \\
\text { time to RRT=32hrs }\end{array}$ & HIGH & $\begin{array}{l}\text { Mortality: Early } 16 / 48 \\
(33 \%) \text { died, } \\
\text { Late } 19 / 52 \text { died; } p=0.74\end{array}$ \\
\hline
\end{tabular}


Table 1 Trial Summary Table by Study Type ( $n=36)$ (Continued)

RCT Totals

Prospective Trials

\begin{tabular}{|c|c|c|c|c|c|c|c|c|c|c|c|c|c|c|}
\hline Liu, 2006 [18] & $\begin{array}{l}\text { Prospective } \\
\text { Observational } \\
\text { Multicentre }\end{array}$ & $\begin{array}{l}\text { Multi } \\
\text { countries }\end{array}$ & $\begin{array}{l}\text { Feb } 1999- \\
\text { Aug } 2001\end{array}$ & GFR<30m//min/1.73m2 & Multisystem & 243 & 122 & 121 & $\begin{array}{l}\text { Early } 54 \\
\text { Late } 58\end{array}$ & NR & $\begin{array}{l}\text { Azotemia defined } \\
\text { by BUN }<76 \mathrm{mg} / \mathrm{dL}\end{array}$ & $\begin{array}{l}\text { Azotemia defined } \\
\text { by BUN }>76 \mathrm{mg} / \mathrm{dL}\end{array}$ & $\begin{array}{l}\text { LOW } \\
\text { NOQA }=6\end{array}$ & $\begin{array}{l}28 \text { d mortality: Early } \\
43 / 122(35 \%) \text { died vs } \\
\text { Late 50/121(41\%) } \\
P=0.09 \text { Favors Early }\end{array}$ \\
\hline $\begin{array}{l}\text { lyem, } \\
2009[19]\end{array}$ & $\begin{array}{l}\text { Prospective } \\
\text { Observational } \\
\text { cohort }\end{array}$ & Turkey & $\begin{array}{l}\text { May } 2004 \text { - } \\
\text { April } 2007\end{array}$ & $\begin{array}{l}\text { Preexisting renal } \\
\text { disease and pre } \\
\text { operative high } \\
\text { levels of urea } \\
\text { and creatinine }\end{array}$ & $\begin{array}{l}\text { Post cardiac } \\
\text { surgery }\end{array}$ & 185 & 95 & 90 & $\begin{array}{l}\text { Early: } 64 ; \\
\text { Late: } 62\end{array}$ & NR & $\begin{array}{l}\text { TIME: Evidence } \\
\text { of } 50 \% \text { increase } \\
\text { in BUN, low urine } \\
\text { output }(<0.5 \mathrm{~mL} / \mathrm{kg} / \mathrm{h}) \\
\text { triggering RRT } \\
\text { started }<48 \mathrm{hrs}\end{array}$ & $\begin{array}{l}\text { TIME }>48 \text { hrs to } \\
\text { start of RRT for similar } \\
\text { markers of renal failure } \\
\text { managed medically for } \\
\text { minimum 48hrs }\end{array}$ & $\begin{array}{l}\text { LOW } \\
\text { NOQA }=7\end{array}$ & $\begin{array}{l}\text { In hosp mortality: Early } \\
\text { 5/95(5\%) died, Late } \\
\text { 6/90(7\%) died; NS }\end{array}$ \\
\hline $\begin{array}{l}\text { Bagshaw, } \\
2009[20]\end{array}$ & $\begin{array}{l}\text { Prospective } \\
\text { Observational } \\
\text { Multicentre } \\
\text { (BEST Kidney) }\end{array}$ & $\begin{array}{l}23 \\
\text { countries }\end{array}$ & $\begin{array}{l}\text { Sept } 2000 \text { - } \\
\text { Dec } 2001\end{array}$ & $\begin{array}{l}\text { Pre existing chronic } \\
\text { RRT, drug toxicity, } \\
\text { age }<12\end{array}$ & Multisystem & 1227 & 959 & 268 & $\begin{array}{l}\text { Early: } 60, \\
\text { Delayed: } 63, \\
\text { Late: } 64 ; \\
p=0.003\end{array}$ & $\begin{array}{l}\text { Early: SOFA 10.9- } \\
\text { SAPS2=53.5 } \\
\text { Delayed: SOFA 11.1- } \\
\text { SAPS2=46 } \\
\text { Late: SOFA 10.7- } \\
\text { SAPS2=43.1; } \\
p=0.04\end{array}$ & $\begin{array}{l}\text { TIME: Early RRT started for } \\
\text { azotemia (Urea>30mmol/L } \\
\text { or low urine output } \times 12 \mathrm{~h}) \\
<2 \mathrm{~d}(n=785) \text {, Delayed } \\
\text { RRT started } 2-5 \mathrm{~d}(n=174) \\
\text { from ICU admission }\end{array}$ & $\begin{array}{l}\text { RRT started } \\
>5 \text { d from ICU } \\
\text { admission }\end{array}$ & $\begin{array}{l}\text { LOW } \\
\text { NOQA }=7\end{array}$ & $\begin{array}{l}\text { Hosp mortality: Early } \\
\text { 462/785(59\%) died, } \\
\text { Delayed 108/174(62\%) } \\
\text { died, Late } 195 / 268(72 \%) \\
\text { died; } P<0.0011 \\
\text { Favors Early }\end{array}$ \\
\hline $\begin{array}{l}\text { Shiao, } \\
2009[21]\end{array}$ & $\begin{array}{l}\text { Prospective } \\
\text { Observational } \\
\text { Multicentre }\end{array}$ & Taiwan & $\begin{array}{l}\operatorname{Jan} 2002- \\
\operatorname{Dec} 2005\end{array}$ & $\begin{array}{l}\text { Prior dialysis, without } \\
\text { surgery, or surgery } \\
\text { did not involve } \\
\text { abdominal cavity. } \\
\text { History of renal trasplant }\end{array}$ & $\begin{array}{l}\text { Major } \\
\text { abdominal } \\
\text { surgery }\end{array}$ & 98 & 51 & 47 & $\begin{array}{l}\text { Early: } 65 ; \\
\text { Late: } 68\end{array}$ & $\begin{array}{l}\text { Early: SOFA 8.3- } \\
\text { APACHE2 }=18.2 ; \\
\text { Late: SOFA 8.5- } \\
\text { APACHE2 }=18.8\end{array}$ & $\begin{array}{l}\text { BIOCHEM: RIFLE criteria: } \\
\text { RISK or pre-RISK criteria } \\
\text { (Mean Time to RRT } \\
\text { from ICU Admit }=7.3 \mathrm{~d} \text { ) }\end{array}$ & $\begin{array}{l}\text { RIFLE criteria: } \\
\text { INJURY or } \\
\text { FAlLURE criteria } \\
\text { (Mean Time to RRT } \\
\text { from ICU Admit=8.4d) }\end{array}$ & $\begin{array}{l}\mathrm{HIGH} \\
\mathrm{NOQA}=7\end{array}$ & $\begin{array}{l}\text { Hosp mortality: Early } \\
22 / 51(43 \%) \\
\text { Late35/47(75\%); } \\
p=0.0028 \text { Favors Early }\end{array}$ \\
\hline $\begin{array}{l}\text { Sabater, } \\
2009[22]\end{array}$ & $\begin{array}{l}\text { Prospective } \\
\text { Observational }\end{array}$ & Spain & 2 years & NR & Multisystem & 148 & 44 & 104 & $\begin{array}{l}\text { All patients } \\
\text { mean }=60 ; \mathrm{NR}\end{array}$ & $\begin{array}{l}\text { Early: } \mathrm{APACHE}=26 ; \\
\text { Late: } \mathrm{APACHE2}=24\end{array}$ & $\begin{array}{l}\text { BIOCHEM: RRT initiated for } \\
\text { RIFLE: RISK \& INJURY; } \\
\text { (Mean RRT start 2.2d } \\
\text { post ICU admit) }\end{array}$ & $\begin{array}{l}\text { RRT initiated for RIFLE: } \\
\text { FAlLURE; (Mean RRT start } \\
6.4 \text { post ICU admit) }\end{array}$ & $\begin{array}{l}\text { LOW } \\
\text { NOQA }=7\end{array}$ & $\begin{array}{l}\text { Mortality: Early 21/44 } \\
\text { died, Late 68/104 died. } \\
P=0.047 \text { Favors Early }\end{array}$ \\
\hline $\begin{array}{l}\text { Elseviers, } \\
2010[23]\end{array}$ & $\begin{array}{l}\text { Prospective } \\
\text { Observational } \\
\text { Multicentre }\end{array}$ & Belgium & 2001-2005 & $\begin{array}{l}\text { Pre existing renal } \\
\text { disease }(C r<1.5 \mathrm{mg} / \mathrm{dl}) \text {, } \\
\text { reduced kidney size on } \\
\text { ultrasound }\end{array}$ & Multisystem & 1303 & 653 & 650 & $\begin{array}{l}\text { Early } 64 ; \\
\text { Late } 67\end{array}$ & $\begin{array}{l}\text { Early: SOFA 9.9- } \\
\text { APACHE2=25.2; } \\
\text { Late: } \text { SOFA 8.5- } \\
\text { APACHE2 }=5.2 \text {; } \\
p=0.001\end{array}$ & $\begin{array}{l}\text { BIOCHEM: Unspecified } \\
\text { SHARF scoring } \\
\text { criteria w/serum } \\
C r>2 \mathrm{mg} / \mathrm{dL} \text {. }\end{array}$ & $\begin{array}{l}\text { Conservative } \\
\text { approach = } \\
\text { No RRT }\end{array}$ & $\begin{array}{l}\text { LOW } \\
\text { NOQA }=5\end{array}$ & $\begin{array}{l}\text { Mortality: Early 379/653 } \\
\text { (58\%) died, Late } 280 / 650 \\
(43 \%) \text { died; } p<0.001 \\
\text { Favors Late }\end{array}$ \\
\hline $\begin{array}{l}\text { Vaara, } \\
2012[24]\end{array}$ & $\begin{array}{l}\text { Prospective } \\
\text { Observational } \\
\text { Multicentre } \\
\text { (FINNAK Study) }\end{array}$ & Finland & $\begin{array}{l}\text { Sep } 2011 \text { - } \\
\text { Feb } 2012\end{array}$ & NR & $\begin{array}{l}\text { Sepsis, } \\
\text { Cardiogenic } \\
\text { Shock }\end{array}$ & 261 & NR & NR & NR & $\begin{array}{l}\text { Survivors: } \\
\text { SAPS2=47; } \\
\text { Non-survivors: } \\
\text { SAPS2 }=66\end{array}$ & $\begin{array}{l}\text { TIME: Time }<24 \text { hrs from } \\
\text { ICU admit }\end{array}$ & $\begin{array}{l}\text { Time> 24hrs } \\
\text { from ICU admit }\end{array}$ & $\begin{array}{l}\text { LOW } \\
\text { NOQA }=5\end{array}$ & $\begin{array}{l}\text { OR for late } 2.69 \\
(1.07-6.73, p=0.035) \text {. } \\
\text { Favors Early }\end{array}$ \\
\hline $\begin{array}{l}\text { Perez, } \\
2012[25]\end{array}$ & $\begin{array}{l}\text { Prospective } \\
\text { Observational }\end{array}$ & Spain & & NR & Sepsis & 244 & 135 & 109 & $\begin{array}{l}\text { Early } 62 ; \\
\text { Late } 62\end{array}$ & $\begin{array}{l}\text { Early: SOFA 12; } \\
\text { Late: SOFA } 11\end{array}$ & $\begin{array}{l}\text { TIME: Time from ICU admission } \\
\text { to RRT < } 48 \mathrm{~h}\end{array}$ & TIME $>48 \mathrm{hrs}$ & $\begin{array}{l}\text { LOW } \\
\text { NOQA }=5\end{array}$ & $\begin{array}{l}90 \text { d mortality: } \\
\text { Early } 71 / 135(53 \%) \text { died, } \\
\text { Late } 78 / 109(72 \%) \text { died; } \\
p=0.003 . \\
\text { Favors Early }\end{array}$ \\
\hline Lim, 2014 [27] & $\begin{array}{l}\text { Single Centre } \\
\text { Prospective Cohort }\end{array}$ & Singapore & $\begin{array}{l}\text { Dec } 2010 \text { - } \\
\text { April } 2013\end{array}$ & $\begin{array}{l}\text { Chronic dialysis } \\
\text { patients, Dialysis } \\
\text { initiated prior to ICU } \\
\text { admission }\end{array}$ & $\begin{array}{l}\text { Medical \& } \\
\text { Surgical } \\
\text { patients }\end{array}$ & 140 & 84 & 56 & $\begin{array}{l}\text { Early } 60 ; \\
\text { Late } 64\end{array}$ & $\begin{array}{l}\text { Early: SOFA 7; } \\
\text { Late: SOFA 11; } \\
p=0.001\end{array}$ & $\begin{array}{l}\text { BIOCHEM: AKIN stage } \\
1 \text { or } 2 \text { AND compelling } \\
\text { indication or AKIN stage } \\
3(\mathrm{C} r \geq 354 \mu \mathrm{mol} / / \text { or } \mathrm{Cr}>300 \%\end{array}$ & $\begin{array}{l}\text { Traditional indications: } \\
\mathrm{K}>6 \mathrm{mmmo} / \mathrm{L} \text {, Urea } \\
\geq 30 \mathrm{mmol} / \mathrm{L}, \mathrm{pH}<7.25, \text { Bicarb }\end{array}$ & $\begin{array}{l}\text { LOW } \\
\text { NOQA }=6\end{array}$ & $\begin{array}{l}\text { Hosp mortality: } \\
\text { Early } 36 / 84(43 \%) \text { died, } \\
\text { Late 37/56(66\%) died; }\end{array}$ \\
\hline
\end{tabular}

Pooled mortality:

Early 120/404 (29.7\%

Late $117 / 382$

$(30.6 \%) ; n=7$

$\begin{array}{lll}786 & 404 & 382\end{array}$




Table 1 Trial Summary Table by Study Type ( $n=36$ ) (Continued)

\begin{tabular}{|c|c|c|c|c|c|c|c|c|c|c|c|c|c|c|}
\hline & & & & & & & & & & & $\begin{array}{l}\text { baseline w/urine }<0.3 \mathrm{cc} / \mathrm{kg} / \mathrm{h} \\
\text { for } 24 \mathrm{~h} \text { or anuria }>12 \mathrm{~h} \text { ) }\end{array}$ & $\begin{array}{l}<10 \mathrm{mmol} / \mathrm{L}, \text { Pulm edema, } \\
\text { Uremic encephalopathy/pericarditis }\end{array}$ & & $\begin{array}{l}p=0.007 \\
\text { Favors Early }\end{array}$ \\
\hline Jun, 2014 [26] & $\begin{array}{l}\text { Nested } \\
\text { Observational, } \\
\text { Multi-Centre Study } \\
\text { 'RENAL' Study Group }\end{array}$ & $\begin{array}{l}\mathrm{NZ}, \\
\text { Australia }\end{array}$ & $\begin{array}{l}\text { Dec } 2005 \text { - } \\
\text { Nov } 2008\end{array}$ & $\begin{array}{l}\text { Age }<18 \text {, Prior } \\
\text { RRTduring admission, } \\
\text { Prior RRT for CKD }\end{array}$ & Sepsis & 439 & 219 & 220 & $\begin{array}{l}\text { Early } 65 ; \\
\text { Late } 64\end{array}$ & $\begin{array}{l}\text { Early: SOFA: } 2.0- \\
\text { APACHE3 }=107 \\
\text { Late: } \text { SOFA 2.1- } \\
\text { APACHE3=100, } \\
\text { P<0.001 }\end{array}$ & $\begin{array}{l}\text { TIME: AKI diagnosis to } \\
\text { randomization < } 17.6 \text { hrs }\end{array}$ & $\begin{array}{l}\text { Time from AKI diagnosis } \\
\text { to randomization }>17.6 \text { hrs }\end{array}$ & $\begin{array}{l}\text { LOW } \\
\text { NOQA }=6\end{array}$ & $\begin{array}{l}28 \text { d mortality: } \\
\text { Early 82/219(37\%) } \\
\text { died; Late } 84 / 220(38 \%) \\
\text { died }(p=0.923) \text { NS }\end{array}$ \\
\hline $\begin{array}{l}\text { PROSPECTIVE } \\
\text { TOTALS }\end{array}$ & & & & & & 4288 & 2362 & 1665 & & & & & & $\begin{array}{l}\text { Pooled mortality: } \\
\text { Early 1229/2362 (52\%), } \\
\text { Late } 833 / 1665 \\
(50 \%) ; n=10\end{array}$ \\
\hline \multicolumn{15}{|c|}{ Retrospective Trials } \\
\hline $\begin{array}{l}\text { Gettings, } \\
1999[28]\end{array}$ & $\begin{array}{l}\text { Retrospective } \\
\text { cohort }\end{array}$ & USA & $1989-1997$ & $\begin{array}{l}\text { CRRT duration } \\
<48 \text { hrs, Pediatric } \\
\text { patients, Incomplete } \\
\text { records }\end{array}$ & Trauma & 100 & 41 & 59 & $\begin{array}{l}\text { Early } 40 ; \\
\text { Late } 48\end{array}$ & $\begin{array}{l}\text { Early ISS }=33.0 ; \\
\text { Late ISS }=37.2\end{array}$ & $\begin{array}{l}\text { BIOCHEM: BUN }<60 \mathrm{mg} / \mathrm{dL} \\
\text { AND Oliguria, Vol overload, } \\
\text { Electrolytes, Uremia; Mean RRT } \\
\text { start post admission } \\
\text { day 10; } p<0.0001\end{array}$ & $\begin{array}{l}\text { BUN > } 60 \text { mg/dL AND Oliguria, } \\
\text { Vol overload, Electrolytes, } \\
\text { Uremia; Mean RRT start post } \\
\text { admission day } 19\end{array}$ & $\begin{array}{l}\text { LOW } \\
\text { NOQA }=5\end{array}$ & $\begin{array}{l}\text { Hosp mortality: } \\
\text { Early } 25 / 41(61 \%) \text { died, } \\
\text { Late } 47 / 59(80 \%) \text { died; } \\
p=0.041 \text { Favors Early }\end{array}$ \\
\hline $\begin{array}{l}\text { Elahi, } \\
2004[29]\end{array}$ & $\begin{array}{l}\text { Retrospective } \\
\text { cohort }\end{array}$ & UK & $\begin{array}{l}\text { Jan } 2002- \\
\operatorname{Jan} 2003\end{array}$ & $\begin{array}{l}\text { Preexisting renal } \\
\text { disease }\end{array}$ & $\begin{array}{l}\text { Post cardiac } \\
\text { surgery }\end{array}$ & 64 & 36 & 28 & $\begin{array}{l}\text { Early } 69 ; \\
\text { Late } 68\end{array}$ & NR & $\begin{array}{l}\text { BIOCHEM: Low } \\
\text { urine output = less than } \\
100 \mathrm{ml} \text { within } 8 \mathrm{~h} \\
\text { after surgery:Mean RRT } \\
\text { start } 0.78 \text { days }\end{array}$ & $\begin{array}{l}\text { Traditional indications: Urea } \\
\geq 30 \mathrm{mmol} / \mathrm{L}, \mathrm{Cr} \text { Elahi, } 2004[29] \\
\geq 250 \mathrm{mmo} / \mathrm{L}, \mathrm{K}>6.0 \mathrm{mEg} / \mathrm{L} ; \\
\text { Mean RRT start } 2.5 \text { days }\end{array}$ & $\begin{array}{l}\text { LOW } \\
\text { NOQA }=6\end{array}$ & $\begin{array}{l}28 \text { d mortality: } \\
\text { Early-8/36 died (22\%), } \\
\text { Late-12/28 (43\%); } \\
p<0.05 \text { Favors Early }\end{array}$ \\
\hline $\begin{array}{l}\text { Demirkilic, } \\
2004 \text { [30] }\end{array}$ & $\begin{array}{l}\text { Retrospective } \\
\text { cohort }\end{array}$ & Turkey & $\begin{array}{l}\text { Mar } 1992- \\
\text { Sep } 2001\end{array}$ & NR & $\begin{array}{l}\text { Post Cardiac } \\
\text { Surgery }\end{array}$ & 61 & 34 & 27 & NR $p=0.3$ & NR & $\begin{array}{l}\text { BIOCHEM: Low } \\
\text { urine output }=\text { less than } \\
100 \mathrm{ml} \text { within } 8 \mathrm{hrs} \\
\text { post op; Mean RRT } \\
\text { start } 0.88 \text { days }\end{array}$ & $\begin{array}{l}\mathrm{Cr} \geq 5 \mathrm{mg} / \mathrm{dL} \text {, or } \mathrm{K}>5.5 \mathrm{mEg} / \mathrm{L} \\
\text { w/med mgmmt; Mar 92-Jun 96; } \\
\text { Mean RRT start } 2.56 \text { days }\end{array}$ & $\begin{array}{l}\text { LOW } \\
\text { NOQA=6 }\end{array}$ & $\begin{array}{l}\text { Hosp mortality: } \\
\text { Early } 8 / 34(23 \%), \\
\text { Late } 15 / 27(56 \%) ; \\
P=0.016 \text { Favors Early }\end{array}$ \\
\hline $\begin{array}{l}\text { Wu, } \\
2007[32]\end{array}$ & $\begin{array}{l}\text { Retrospective } \\
\text { cohort }\end{array}$ & Taiwan & $\begin{array}{l}\text { July 2002- } \\
\text { Jan2005 }\end{array}$ & $\begin{array}{l}\text { Hepatorenal } \\
\text { syndrome } \\
\text { from cirrhosis, } \\
\text { liver trasplant, } \\
\text { cardiopolmunary } \\
\text { resuccitation }\end{array}$ & $\begin{array}{l}\text { Acute liver } \\
\text { failure }\end{array}$ & 80 & 54 & 26 & $\begin{array}{l}\text { Early } 55 ; \\
\text { Late } 63 ; p=0.03\end{array}$ & $\begin{array}{l}\text { Early: SOFA 12.4- } \\
\text { APACHE2 }=18.2 ; \\
\text { Late: SOFA 13.2- } \\
\text { APACHE2 } 20.5\end{array}$ & $\begin{array}{l}\text { BIOCHEM: BUN } \\
<80 \mathrm{mg} / \mathrm{dL} \text { AND } \\
\text { traditional indications } \\
\text { present }\end{array}$ & $\begin{array}{l}\text { Traditional indications present } \\
\text { with BUN > 80mg/dL }\end{array}$ & $\begin{array}{l}\text { LOW } \\
\text { NOQA }=6\end{array}$ & $\begin{array}{l}30 \text { d mortality: } \\
\text { Early 34/54(63\%) died vs } \\
\text { Late 22/26(85\%) died; } \\
P=0.04 \text { Favors Early }\end{array}$ \\
\hline $\begin{array}{l}\text { Andrade, } \\
2007[31]\end{array}$ & $\begin{array}{l}\text { Retrospective } \\
\text { cohort }\end{array}$ & Brazil & $2002-2005$ & $\begin{array}{l}\text { Patients who did } \\
\text { not have both AKI and } \\
\text { respiratory failure believed } \\
\text { secondary to leptospirosis }\end{array}$ & Leptospirosis & 33 & 18 & 15 & $\begin{array}{l}\text { Early } 42 ; \\
\text { Late } 44\end{array}$ & $\begin{array}{l}\text { Early: APACHE2 }=24.5 ; \\
\text { Late: } A \text { APACHE2 }=26\end{array}$ & $\begin{array}{l}\text { TIME: Mean time } \\
\text { to RRT }=265 \mathrm{~min}\end{array}$ & Mean time to $R R T=1638 \mathrm{~min}$ & $\begin{array}{l}\text { LOW } \\
\text { NOQA }=5\end{array}$ & $\begin{array}{l}\text { Hosp mortality: } \\
\text { Early } 3 / 18(17 \%) \text { died, } \\
\text { Late } 10 / 15(67 \%) \text { died; } \\
P=0.01 \text { Favors Early }\end{array}$ \\
\hline $\begin{array}{l}\text { Manche, } \\
2008[33]\end{array}$ & $\begin{array}{l}\text { Retrospective } \\
\text { cohort }\end{array}$ & Malta & $1995-2006$ & NR & $\begin{array}{l}\text { Post Cardiac } \\
\text { Surgery }\end{array}$ & 71 & 56 & 15 & $\begin{array}{l}\text { Early 66; } \\
\text { Late 63 }\end{array}$ & NR & $\begin{array}{l}\text { BIOCHEM: Urine } \\
\text { output }<0.5 \mathrm{~mm} / \mathrm{kg} / \mathrm{hr} \\
\text { unresponsive to } \\
\text { med mgmt; Mean } \\
\text { RRT start 8.6hrs } \\
\text { post-op }\end{array}$ & $\begin{array}{l}\text { Oliguria (output }<0.5 \mathrm{~mm} / \mathrm{kg} / \mathrm{hr} \text { ) } \\
\text { refractory to med mgmt; Mean } \\
\text { RRT start } 41.2 \mathrm{hrs} \text { post-op }\end{array}$ & $\begin{array}{l}\text { LOW } \\
\text { NOQA }=6\end{array}$ & $\begin{array}{l}\text { Mortality: } \\
\text { Early 14/56(25\%) died, } \\
\text { Late } 13 / 15(87 \%) \text { died; } \\
P=0.0000125 \text { Favors Early }\end{array}$ \\
\hline $\begin{array}{l}\text { Lundy, } \\
2009[34]\end{array}$ & $\begin{array}{l}\text { Retrospective } \\
\text { cohort }\end{array}$ & us & $\begin{array}{l}\text { Nov } 2005 \text { - } \\
\text { Aug } 2007\end{array}$ & $\begin{array}{l}\text { Preexisting renal } \\
\text { disease, burn size } \\
\text { of less than } 40 \% \\
\text { Non-thermal injury, } \\
\text { lithium toxicity }\end{array}$ & $\begin{array}{l}\text { Severe } \\
\text { Burned } \\
\text { patients }\end{array}$ & 57 & 29 & 28 & $\begin{array}{l}\text { Early } 27 ; \\
\text { Late } 38 \mathrm{P}=0.06\end{array}$ & $\begin{array}{l}\text { Early: SOFA 13- } \\
\text { APACHE2=35; } \\
\text { Late: SOFA 13- } \\
\text { APACHE2 }=36\end{array}$ & $\begin{array}{l}\text { BIOCHEM: AKIN stage } \\
2(+ \text { shock)/3; } \\
\text { Mean time from admit } \\
\text { to RRT }=17 \text { days }\end{array}$ & $\begin{array}{l}\text { Mean time from admit to } \\
\text { AKIN stage } 2(+ \text { shock }) / 3 \text { but } \\
\text { not dialyzed }=23 \text { days }\end{array}$ & $\begin{array}{l}\text { LOW } \\
\text { NOQA }=6\end{array}$ & $\begin{array}{l}28 \text { d mortality: } \\
\text { Early } 9 / 29(31 \%) \text { died, } \\
\text { Late } 24 / 28(85 \%) \text { died; } \\
P<0.002 \text {; Favors Early }\end{array}$ \\
\hline
\end{tabular}


Table 1 Trial Summary Table by Study Type $(n=36)$ (Continued)

\begin{tabular}{|c|c|c|c|c|c|c|c|c|c|c|c|c|c|c|}
\hline $\begin{array}{l}\text { Carl, } \\
2010[35]\end{array}$ & $\begin{array}{l}\text { Retrospective } \\
\text { cohort }\end{array}$ & us & 2000-2004 & $\begin{array}{l}\text { Baseline eGFOR } \\
<30 \mathrm{ml} / \mathrm{min} \text {, Age } \\
<18 \text { \& prisoners }\end{array}$ & Sepsis & 147 & 85 & 62 & $\begin{array}{l}\text { Early } 52 ; \\
\text { Late } 56\end{array}$ & $\begin{array}{l}\text { Early: APACHE2 }=24.8 ; \\
\text { Late: APACHE2 } 24.7\end{array}$ & $\begin{array}{l}\text { BIOCHEM: BUN } \\
<100 \mathrm{mg} / \mathrm{dL}+\text { AKIN stage }>2 ; \\
\text { Mean ICU stay prior } \\
\text { to } \mathrm{RRT}=6.3 \text { days }\end{array}$ & $\begin{array}{l}\text { BUN }>100 \mathrm{mg} / \mathrm{dL}+\text { AKIN stage } \\
>2 \text {; Mean ICU stay prior } \\
\text { to RRT } 12.3 \text { days }\end{array}$ & $\begin{array}{l}\text { HIGH } \\
\text { NOQA }=7\end{array}$ & $\begin{array}{l}28 \text { d mortality: } \\
\text { Early 44/85(52\%) died, } \\
\text { Late 42/62(68\%); } \\
P<0.05 \text { Favors Early }\end{array}$ \\
\hline $\begin{array}{l}\text { Chou, } \\
2011[37]\end{array}$ & $\begin{array}{l}\text { Retrospective } \\
\text { cohort 'NSARF' } \\
\text { database }\end{array}$ & Taiwan & $\begin{array}{l}\text { Jan } 2002- \\
\text { Oct } 2009\end{array}$ & $\begin{array}{l}\text { Age } 18 \text {, ICU stay } \\
<2 \text { days, RRT }<2 \text { days }\end{array}$ & Sepsis + AKI & 370 & 192 & 178 & $\begin{array}{l}\text { Early 64; } \\
\text { Late 66 }\end{array}$ & $\begin{array}{l}\text { Early: SOFA } 10.8- \\
\text { APACHE2 }=12.3 ; \\
\text { Late: SOFA } 11.6- \\
\text { APACHE2 }=14.0\end{array}$ & $\begin{array}{l}\text { BIOCHEM: RIFLE criteria: } \\
\text { RISK or } \\
\text { pre-RISK criteria }\end{array}$ & $\begin{array}{l}\text { RIFLE criteria: INJURY } \\
\text { or FAlLURE criteria }\end{array}$ & $\begin{array}{l}\text { LOW } \\
\text { NOQA }=6\end{array}$ & $\begin{array}{l}\text { Hosp mortality: } \\
\text { Early 135/192(71\%) } \\
\text { died, Late 124/178 (70\%) } \\
\text { died }(P=0.98)\end{array}$ \\
\hline $\begin{array}{l}\text { Vats, } \\
2011 \text { [38] }\end{array}$ & $\begin{array}{l}\text { Retrospective } \\
\text { cohort }\end{array}$ & USA & $\begin{array}{l}\text { Jan1 } 1999 \text { - } \\
\text { Feb } 2006\end{array}$ & $\begin{array}{l}\text { Renal transplant, } \\
\text { Pre-morbid ESRD } \\
\text { on dialysis, RRT } \\
<24 h \text {, insufficient data }\end{array}$ & Multisystem & 230 & $\mathrm{NR}$ & NR & $\begin{array}{l}\text { All patients } \\
\text { mean }=66 \mathrm{NR}\end{array}$ & NR & $\begin{array}{l}\text { TIME: Time from AKI to } \\
\text { RRT < } 6 \text { days }\end{array}$ & Time from AKI to RRT $26 \mathrm{~d}$ & $\begin{array}{l}\text { LOW } \\
\text { NOQA }=5\end{array}$ & $\begin{array}{l}\text { OR for Late Mortality } \\
(>6 d) 11.66(1.26-107.9) \\
P=0.0305 \text {, Favors Early }\end{array}$ \\
\hline $\mathrm{Ji}, 2011$ [36] & $\begin{array}{l}\text { Retrospective } \\
\text { cohort }\end{array}$ & China & $\begin{array}{l}\text { Ap } 2004 \text { - } \\
\text { Mar } 2009\end{array}$ & $\begin{array}{l}\text { Patients readmitted } \\
\text { post discharge, } \\
\text { Discharged } \\
\text { against medical } \\
\text { advice, Death <24hrs }\end{array}$ & $\begin{array}{l}\text { Post cardiac } \\
\text { surgery }\end{array}$ & 58 & 34 & 24 & $\begin{array}{l}\text { Early } 64 ; \\
\text { Late } 62\end{array}$ & $\begin{array}{l}\text { Early: APACHE3=69.3; } \\
\text { Late: APACHE3 }=88.2 \\
p<0.001\end{array}$ & $\begin{array}{l}\text { TIME: Time from urine } \\
\text { output }<0.5 \mathrm{~m} / \mathrm{kg} / \mathrm{h} \\
\text { to RRT } 12 \mathrm{~h} \text {; } \\
\text { Mean oliguria to } \\
\text { start of RRT } 8.4 \mathrm{hrs}\end{array}$ & $\begin{array}{l}\text { Urine output }<0.5 \mathrm{~m} / \mathrm{kg} / \mathrm{h} \text { \& } \\
\text { Time to RRT }>12 \mathrm{~h} \text { post oliguria; } \\
\text { Mean oliguria to start of RRT } \\
\text { 21.5hrs }\end{array}$ & $\begin{array}{l}\text { LOW } \\
\text { NOQA }=6\end{array}$ & $\begin{array}{l}\text { Hosp mortality: Early } \\
3 / 34(9 \%) \text { died, } \\
\text { Late } 9 / 24(37 \%) \text {; } \\
p=0.02 \text { Favors Early }\end{array}$ \\
\hline $\begin{array}{l}\text { Shiao, } \\
2012[41]\end{array}$ & $\begin{array}{l}\text { Retrospective } \\
\text { cohort 'NSARF' } \\
\text { database }\end{array}$ & Taiwan & $\begin{array}{l}\text { Jan } 2002 \text { - } \\
\text { Apr } 2009\end{array}$ & $\begin{array}{l}\text { Dialysis before } \\
\text { surgery, ESRD }\end{array}$ & Surgical & 648 & 436 & 212 & $\begin{array}{l}\text { Early 62; } \\
\text { Late 66; } P=0.009\end{array}$ & $\begin{array}{l}\text { Early: SOFA 11.4- } \\
\text { APACHE2 }=12.7 \\
\text { Late: SOFA 11.3- } \\
\text { APACHE2 } 2=12.8\end{array}$ & $\begin{array}{l}\text { TIME: Time to development } \\
\text { of tradtional RRT } \\
\text { indications < } 3 \text { d; Mean } \\
\text { time to start of RRT } 1.4 \text { days }\end{array}$ & $\begin{array}{l}\text { Traditional RRT indications } \\
\text { AND start of RRT > } 3 \text { days; } \\
\text { Mean time to start of RRT } \\
\text { 18days }\end{array}$ & $\begin{array}{l}\text { LOW } \\
\text { NOQA }=6\end{array}$ & $\begin{array}{l}\text { Hosp mortality: } \\
\text { Early 236/436 (54\%) } \\
\text { died, Late } 143 / 212 \text { (67\%) } \\
\text { died; } P=0.001 \\
\text { Favors Early }\end{array}$ \\
\hline $\begin{array}{l}\text { Chon, } \\
2012[40]\end{array}$ & $\begin{array}{l}\text { Retrospective } \\
\text { cohort }\end{array}$ & $\begin{array}{l}\text { South } \\
\text { Korea }\end{array}$ & $\begin{array}{l}\text { Apr } 2009 \text { - } \\
\text { Oct } 2010\end{array}$ & $\begin{array}{l}\text { Liver cirrhosis, } \\
\text { Pre existing chronic }\end{array}$ & Sepsis & 55 & 36 & 19 & $\begin{array}{l}\text { Early 63; } \\
\text { Late 62 }\end{array}$ & $\begin{array}{l}\text { Early: SOFA 13.5- } \\
\text { APACHE2= 28.7; } \\
\text { Late: SOFA 12- } \\
\text { APACHE2 } 2=28.3\end{array}$ & $\begin{array}{l}\text { TIME: Time to RIFLE } \\
\text { 'Injury'/Failure' } \\
<24 \text { hrs; Mean time to } \\
\text { RRT }=12.5 \text { hrs }\end{array}$ & $\begin{array}{l}\text { Time to RIFLE 'Injury'/Failure' } \\
>24 \mathrm{hrs} ; \text {; Mean time to } \\
\text { RRT }=42.2 \mathrm{hrs}\end{array}$ & $\begin{array}{l}\text { HIGH } \\
\text { NOQA }=7\end{array}$ & $\begin{array}{l}28 \mathrm{~d} \text { mortality: } \\
\text { Early } 7 / 36(38 \%), \\
\text { Late } 9 / 19(47 \%) ; \\
P=0.03 \text { Favors Early }\end{array}$ \\
\hline $\begin{array}{l}\text { Boussekey, } \\
2012 \text { [39] }\end{array}$ & $\begin{array}{l}\text { Retrospective } \\
\text { cohort }\end{array}$ & France & $\begin{array}{l}\operatorname{Jan} 2008 \text { - } \\
\text { Dec } 2010\end{array}$ & $\begin{array}{l}\text { Early trasfer } \\
\text { to another unit }\end{array}$ & Multisystem & 110 & 67 & 43 & $\begin{array}{l}\text { Early 62; } \\
\text { Late } 66\end{array}$ & $\begin{array}{l}\text { Early: SOFA: 11.1- } \\
\text { SAPS2=70; } \\
\text { Late: SOFA } 8.8- \\
\text { SAPS2=57; } p=0.002\end{array}$ & $\begin{array}{l}\text { TIME: Time from } \\
\text { RIFLE- 'Injury' to RRT } \\
<16 \mathrm{hrs} \text {; Mean time } \\
\text { to RRT=6hrs }\end{array}$ & $\begin{array}{l}\text { Time from RilLLE-'Injury' to } \\
\text { RRT > 16hrs; Mean time to } \\
\text { RRT=64hrs }\end{array}$ & $\begin{array}{l}\text { LOW } \\
\text { NOQA }=7\end{array}$ & $\begin{array}{l}28 \text { d mortality: } \\
\text { Early-28/67 (41\%), } \\
\text { Late- } 28 / 43(65 \%) ; \\
P=0.0425 \text { Favors Early }\end{array}$ \\
\hline $\begin{array}{l}\text { Suzuki, } \\
2013[43]\end{array}$ & $\begin{array}{l}\text { Retrospective } \\
\text { cohort }\end{array}$ & Japan & $\begin{array}{l}\text { Jan } 2009 \text { - } \\
\text { Feb } 2013\end{array}$ & $\begin{array}{l}<18, \text { RRT for } \\
\text { ESRD }\end{array}$ & $\begin{array}{l}\text { Sepsis, } \\
\text { Cardiogenic } \\
\text { Shock }\end{array}$ & 189 & 52 & 137 & $\begin{array}{l}\text { All patients } \\
\text { mean }=72 \mathrm{NR}\end{array}$ & $\begin{array}{l}\text { All patients } \\
\text { SAPS II Mean= } 57\end{array}$ & BIOCHEM: RIFLE 'Risk' & RIFLE 'njury' or 'Failure' & $\begin{array}{l}\text { LOW } \\
\text { NOQA }=6\end{array}$ & $\begin{array}{l}\text { Early: OR 0.361 } \\
(95 \% \text { Cl } 0.17-0.78) \text {; } \\
P=0.009 \text {, Favors Early }\end{array}$ \\
\hline $\begin{array}{l}\text { Shum, } \\
2013[43]\end{array}$ & $\begin{array}{l}\text { Retrospective } \\
\text { cohort }\end{array}$ & China & $\begin{array}{l}\operatorname{Jan} 2008- \\
\operatorname{Jun} 2011\end{array}$ & $\begin{array}{l}\text { Age }<18 \text {, Chronic } \\
\text { dialysis, RRT } \\
\text { prior to ICU }\end{array}$ & Sepsis & 120 & 31 & 89 & $\begin{array}{l}\text { qEarly } 74 ; \\
\text { Late } 73\end{array}$ & $\begin{array}{l}\text { Early: SOFA 12- } \\
\text { APACHE4=119; } \\
\text { Late: SOFA 13- } \\
\text { APACHE4=133; } \\
P=0.011\end{array}$ & $\begin{array}{l}\text { BIOCHEM: SRIFLE-'pre- Risk' or } \\
\text { 'Risk' criteria; } \\
\text { Mean time from } \\
\text { ICU admit to } \\
\text { RRT }=20.7 \mathrm{hrs}, P=0.056\end{array}$ & $\begin{array}{l}\text { SRIFLE 'Injury' or 'Failure' criteria; } \\
\text { Mean time from ICU admit } \\
\text { to RRT }=10.8 \text { hrs }\end{array}$ & $\begin{array}{l}\text { LOW } \\
\text { NOQA }=6\end{array}$ & $\begin{array}{l}28 \text { d mortality: } \\
\text { Early-15/31 died } \\
(48.4 \%), \text { Late- } 43 / 89 \\
\text { died (48.3\%); } P=0.994\end{array}$ \\
\hline \multirow[t]{2}{*}{$\begin{array}{l}\text { Tian, } \\
2014[46]\end{array}$} & $\begin{array}{l}\text { Retrospective } \\
\text { cohort }\end{array}$ & China & $\begin{array}{l}\text { Nov } 2009 \text { - } \\
\text { Dec } 2011\end{array}$ & $\begin{array}{l}\text { Age }<12 \text {, Chronic } \\
\text { renal disease, } \\
\text { Terminal illness, } 0 \\
\text { Pre-admit CRRT, } \\
\text { ICU stay }<72 \text { hrs }\end{array}$ & $\begin{array}{l}\text { Sepsis - } \\
\text { AKIN } 1\end{array}$ & 49 & 23 & 26 & $\begin{array}{l}\text { Early } 48 ; \\
\text { Control } 54\end{array}$ & $\begin{array}{l}\text { Early: SOFA 7.6- } \\
\text { APACHE2=12.9; } \\
\text { Control: SOFA } 8.4- \\
\text { APACHE2 }=15.3\end{array}$ & $\begin{array}{l}\text { BIOCHEM: AKIN } 1 \\
\text { (Cr } \geq 26.4 \mu \mathrm{mol} / \mathrm{L} \\
\text { or }>150-200 \% \text { baseline } \\
\& \text { urine }<0.5 \mathrm{cc} / \mathrm{kg} / \mathrm{h} \\
\text { for }>6 \mathrm{~h})\end{array}$ & $\begin{array}{l}\text { No RRT (Control): Patients refused } \\
\text { CRRT for "personal reasons" }\end{array}$ & $\begin{array}{l}\text { LOW } \\
\text { NOQA }=6\end{array}$ & $\begin{array}{l}28 \text { d mortality: } \\
\text { Early 5/23(22\%) died, } \\
\text { Control 11/26 (42\%) } \\
\text { died (NS) }\end{array}$ \\
\hline & & & & & $\begin{array}{l}\text { Sepsis - } \\
\text { AKIN } 2\end{array}$ & 52 & 31 & 21 & $\begin{array}{l}\text { Early 54; } \\
\text { Control } 61\end{array}$ & $\begin{array}{l}\text { Early: SOFA 9.3- } \\
\text { APACHE2=19; } \\
\text { Control SOFA } 9.6- \\
\text { APACHE2 }=18.3\end{array}$ & $\begin{array}{l}\text { AKIN } 2 \text { (Cr }>200-300 \% \\
\text { baseline \& urine } \\
<0.5 \mathrm{cc} / \mathrm{kg} / \mathrm{h} \text { for }>12 \mathrm{~h})\end{array}$ & $\begin{array}{l}\text { No RRT (Control): Patients refused } \\
\text { CRRT for "personal reasons" }\end{array}$ & & $\begin{array}{l}28 \text { d mortality: } \\
\text { Early } 12 / 31 \text { (39\%) } \\
\text { died, Control 14/21 (67\%) } \\
\text { died; } P<0.05 \text { Favors Early }\end{array}$ \\
\hline
\end{tabular}


Table 1 Trial Summary Table by Study Type ( $n=36)$ (Continued)

\begin{tabular}{|c|c|c|c|c|c|c|c|c|c|c|c|c|c|c|}
\hline & & & & & $\begin{array}{l}\text { Sepsis - } \\
\text { AKIN } 3\end{array}$ & 59 & 46 & 13 & $\begin{array}{l}\text { Early } 50 ; \\
\text { Control } 55\end{array}$ & $\begin{array}{l}\text { Early SOFA } 10- \\
\text { APACHE2=21.8; } \\
\text { Control SOFA } 11.2- \\
\text { APACHE2=20.5 }\end{array}$ & $\begin{array}{l}\text { AKIN } 3(\mathrm{Cr} \geq 3544 \mathrm{~mol} / \mathrm{L} \text { or } \\
\mathrm{Cr}>300 \% \text { baseline w/urine } \\
<0.3 \mathrm{cc} / \mathrm{kg} / \mathrm{h} \text { for } \\
24 \mathrm{~h} \text { or anuria }>12 \mathrm{~h})\end{array}$ & $\begin{array}{l}\text { No RRT (Control): } \\
\text { Patients refused } \\
\text { CRRT for } \\
\text { "personal reasons" }\end{array}$ & & $\begin{array}{l}28 \text { d mortality: } \\
\text { Early } 31 / 46(67 \%) \text { died, } \\
\text { Control } 11 / 13(85 \%) \\
\text { died; NS }\end{array}$ \\
\hline $\begin{array}{l}\text { Serpytis, } \\
2014[45]\end{array}$ & $\begin{array}{l}\text { Retrospective } \\
\text { cohort }\end{array}$ & Lithuania & 2007-2011 & NR & Sepsis & 85 & 42 & 43 & $\begin{array}{l}\text { All patients } \\
\text { mean }=72 \mathrm{NR}\end{array}$ & NR & $\begin{array}{l}\text { TIME: Time from } \\
\text { anuria to RRT }<12 \text { hrs }\end{array}$ & $\begin{array}{l}\text { Time from anuria to } \\
\text { RRT > } 12 \text { hrs }\end{array}$ & $\begin{array}{l}\text { LOW } \\
\text { NOQA }=5\end{array}$ & $\begin{array}{l}\text { Mortality: Early 30/42 } \\
\text { (71\%) died, Late } \\
\text { 39/43(91\%) died; } \\
\text { p=0.028; Favors Early }\end{array}$ \\
\hline $\begin{array}{l}\text { Gaudry, } \\
2014[44]\end{array}$ & $\begin{array}{l}\text { Retrospective } \\
\text { cohort }\end{array}$ & France & $\begin{array}{l}\text { Jan } 2004 \text { - } \\
\text { Nov } 2011\end{array}$ & $\begin{array}{l}\text { Age }<18 \text {, limitation } \\
\text { in medical therapy, } \\
\text { death } 24 \text { hrs, chronic } \\
\text { renal insufficiency, } \\
\text { RRT prior to ICU, } \\
\text { kidney transplant, } \\
\text { lithium toxicity, } \\
\text { multiple myeloma }\end{array}$ & Sepsis & 203 & 91 & 112 & $\begin{array}{l}\text { Early } 65 ; \\
\text { Late } 65\end{array}$ & $\begin{array}{l}\text { Early: SOFA 9- } \\
\text { SAPS2=60; } \\
\text { Control SOFA 8- } \\
\text { SAPS2 }=55, P<0.01\end{array}$ & $\begin{array}{l}\text { BIOCHEM: RRT criteria: } \\
\text { Cr } \geq 300 \mu \mathrm{mol} / \mathrm{L}, \\
\text { Urea }>25 \mathrm{mmmo} / \mathrm{L}, \\
\mathrm{K}>6.5 \mathrm{mmol} / \mathrm{L}, \\
\mathrm{pH}<7.2, \text { Oliguria, } \\
\text { Vol overload, }\end{array}$ & $\begin{array}{l}\text { No RRT initiated/Criteria } \\
\text { not met for RRT }\end{array}$ & $\begin{array}{l}\text { LOW } \\
\text { NOQA }=5\end{array}$ & $\begin{array}{l}\text { Hosp Mortality: } \\
\text { Early 44/91(48\%) died, } \\
\text { Control (No RRT) 29/112 } \\
\text { (26\%) died; P<0.001 } \\
\text { Favors no RRT }\end{array}$ \\
\hline $\begin{array}{l}\text { Retrospective } \\
\text { TOTALS }\end{array}$ & & & & & & 2841 & 1434 & 1177 & & & & & & $\begin{array}{l}\text { Pooled mortality: } \\
\text { Early } 714 / 1434(50 \%), \\
\text { Late } 732 / 1177 \\
(62.2 \%) ; n=19\end{array}$ \\
\hline
\end{tabular}


outcomes, including ICU length of stay (LOS) and hospital LOS. Secondary outcomes were not consistently reported for all studies, and only studies with applicable data were included in our pooled analysis. Weighted means were calculated as a product of the number of patients and mean duration to reach a total and represented as a total of patient-days per study. These values were summed and divided by the total number of patients from all included studies to reach weighted mean duration of LOS for both hospital and ICU LOS metrics. A similar process was used to derive the mean weighted illness severity scores. Other potentially relevant secondary outcomes, including mechanical ventilation requirements, vasopressor requirements, and renal recovery rates, were considered, but these variables were inconsistently reported and commonalities could not be reached among the heterogeneous parameters that were available.

\section{Definition of "early" versus "late"}

Early was defined on the basis of criteria used by the original authors in their respective studies. We accepted a broad definition of early based on biochemical markers according to RIFLE classifications (risk, injury, failure, loss of function, and end-stage kidney disease), Acute Kidney Injury Network (AKIN) stages, or time-based cutoffs (e.g., within a defined time from ICU admission or development of a biochemical "start time"). Accepting a broad definition of early was intended to optimize the potential for identifying an effect associated with "early" RRT. A limitation of this approach is that "early" according to one study investigator might be considered "late" by another study investigator. "Late" RRT criteria involved either usual practice or expectant care (i.e., no RRT initiated). "Usual practice" generally involved implementing RRT following the development of classic RRT indications unresponsive to medical management.

\section{Statistical analysis}

The quality of cohort trials was assessed using the NOQA Scale (range from 0 to 9 , with 9 indicating the highest quality) [11]. The NOQA Scale for cohort studies assesses the domains of population selection, comparability of cohorts, and outcome assessment. A meta-analysis was conducted using the high-quality studies to calculate the pooled OR for mortality at 1 month. A random effects model was used because of the significant heterogeneity between studies on this topic. A random effects model is indicated when study populations differ in ways that could impact the results. Heterogeneity was assessed on the basis of the $Q$ value and $I^{2}$ and $\tau^{2}$ statistics. A $p$ value less than 0.05 was considered statistically significant. All analyses were performed using Comprehensive Meta-Analysis version 3.3.070 software (www.meta-analysis.com; Biostat, Englewood, NJ, USA).

\section{Results}

The systematic literature search yielded 2405 references that were subsequently refined to 36 studies eligible for inclusion in this meta-analysis (see Additional file 3: Figure S2 for article selection breakdown). These references included 7 RCTs [7, 12-17], 10 prospective cohort studies [18-27], and 19 retrospective cohort studies [28-46]. Only nine studies met our criteria for high quality $[7,12,14-17,21,35,40]$. A summary of the fundamental characteristics of all evaluated studies is provided in Table 1.

\section{Primary outcome}

The observed pooled crude mortality rates varied significantly between the high- and low-quality studies. Among the high-quality studies, the pooled "early" RRT study group mortality rate was $34.6 \%$ (192 of 555) compared with $40.2 \%$ (196 of 487) in the pooled "late" RRT group. The low-quality studies demonstrated a pooled "early" RRT group mortality rate of $51.3 \%$ (1871 of 3645) compared with $54.3 \%$ (1486 of 2737) in the "late" RRT groups. The most frequently reported measurement of illness severity in the studies we analyzed was the Sequential Organ Failure Assessment (SOFA) score. The SOFA score has been correlated with critical care patient outcomes [47, 48], but it is not as robust as other scoring systems validated in predicting survival (e.g., Acute Physiology and Chronic Health Evaluation II [APACHE2] or Simplified Acute Physiology Score II [SAPS2]) [49]. The mean weighted SOFA scores in the high-quality studies were 10.2 and 10.4 in the "early" and "late" groups, respectively. SOFA scores were reported for $78 \%$ of patients in the high-quality studies. Among the high-quality studies, the SOFA score appeared to correspond with an APACHE2 score of approximately 20 or a SAPS2 score of approximately 53 when these additional illness severity metrics were reported by the principal investigators. Unfortunately, more detailed quantitative evaluation of illness severity using APACHE2 or SAPS2 scores was not possible, owing to heterogeneous reporting methods between investigators and a lack of sufficient data. SOFA scores were reported for $65 \%$ of the patients in the studies assigned low-quality ratings. The mean weighted SOFA scores in the "early" and "late" groups among the lowquality studies were comparable to those for the highquality studies at 10.0 and 9.2, respectively. No further comments can be made regarding illness severity scores among the low-quality studies, owing to lack of homogeneous and sufficient data. Illness severity scores for all studies are summarized in Table 1.

Pooled analysis of the high-quality studies $(n=9)$ indicates no mortality benefit with "early" versus "late" RRT, with an OR of 0.665 (95\% CI 0.384-1.153, $p=0.146$ ) (Fig. 1). The bulk of the data in support of "early" RRT 


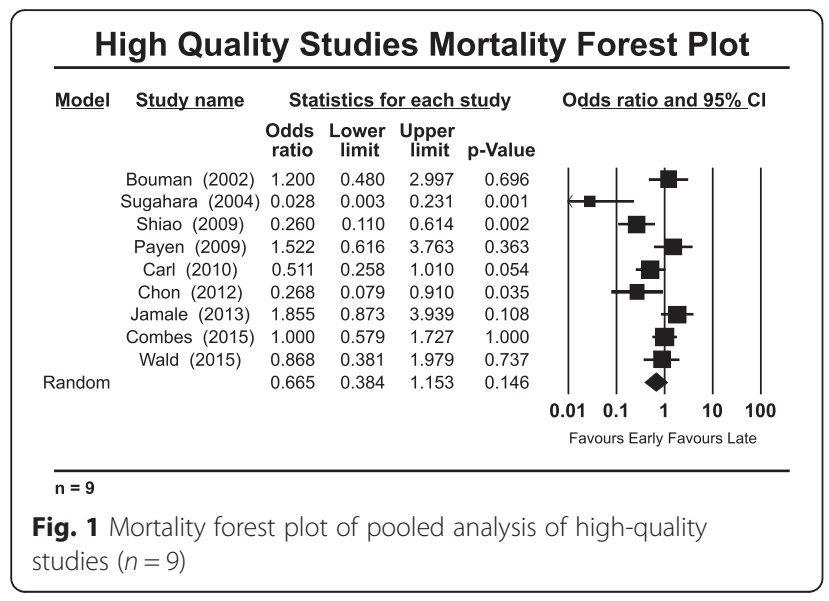

rests in the pooled low-quality studies $(n=27)$, with an OR of 0.471 (95\% CI 0.343-0.649, $p<0.001$ ) (Fig. 2). Similarly to authors of previous meta-analyses, we found very high heterogeneity among studies on this topic. Heterogeneity was highest among the low-quality studies, reflected by a $Q$ value of $163.8, I^{2}$ value of $84 \%$, and $\tau^{2}=0.495(p<0.001)$. Among the high-quality studies, there continued to be statistically significant heterogeneity, with a $Q$ value of $29.1, I^{2}$ value of $72.5 \%$, and $\tau^{2}=$ $0.481(p<0.001)$. Subgroup analysis of the high-quality studies according to ICU admission type and surgical $[14,16,21]$ versus mixed medical admissions $[7,12$, $15,17,35,40]$ demonstrated no significant subgroup mortality benefits associated with "early" RRT (see
Additional file 4: Figure S3a and b for forest plots by ICU admission type). Subgroup analysis among the high-quality studies was also conducted using the definition of early according to time criteria (hours or days) versus biochemical parameters (i.e., rising creatinine, uremia, oliguria) (see Additional file 5: Figure S4a and b for forest plots by biochemical or time definition of early). There were no significant effects observed in pooled mortality trends in studies that defined early by time criteria rather than on the basis of biochemical parameters.

\section{Secondary outcomes}

The secondary outcomes analyzed included ICU LOS and hospital LOS. Five of the nine high-quality studies reported ICU LOS data [12, 16, 17, 35, 40]. The mean weighted ICU LOS in the "early" group was 9.4 days $(n=351)$, compared with 10.8 days $(n=281)$ in the "late" group. None of the studies reported a significant finding with respect to ICU LOS and "early" RRT. Pooled analysis for ICU LOS also demonstrated no significant change in ICU LOS associated with "early" RRT, with a standard difference in the means of -0.035 ( $95 \% \mathrm{CI}-0.196$ to 0.127 , $p=0.674)$ using a fixed effects model $(Q=0.598, p=$ 0.963) (Fig. 3). Hospital LOS was reported in five of nine high-quality studies $[12,16,17,21,40]$. The mean weighted hospital LOS in the "early" group was 19.3 days ( $n=317)$, compared with 17.1 days $(n=266)$ in the "late" group. The pooled hospital LOS data do not reveal any significant difference in hospital LOS using a fixed effects

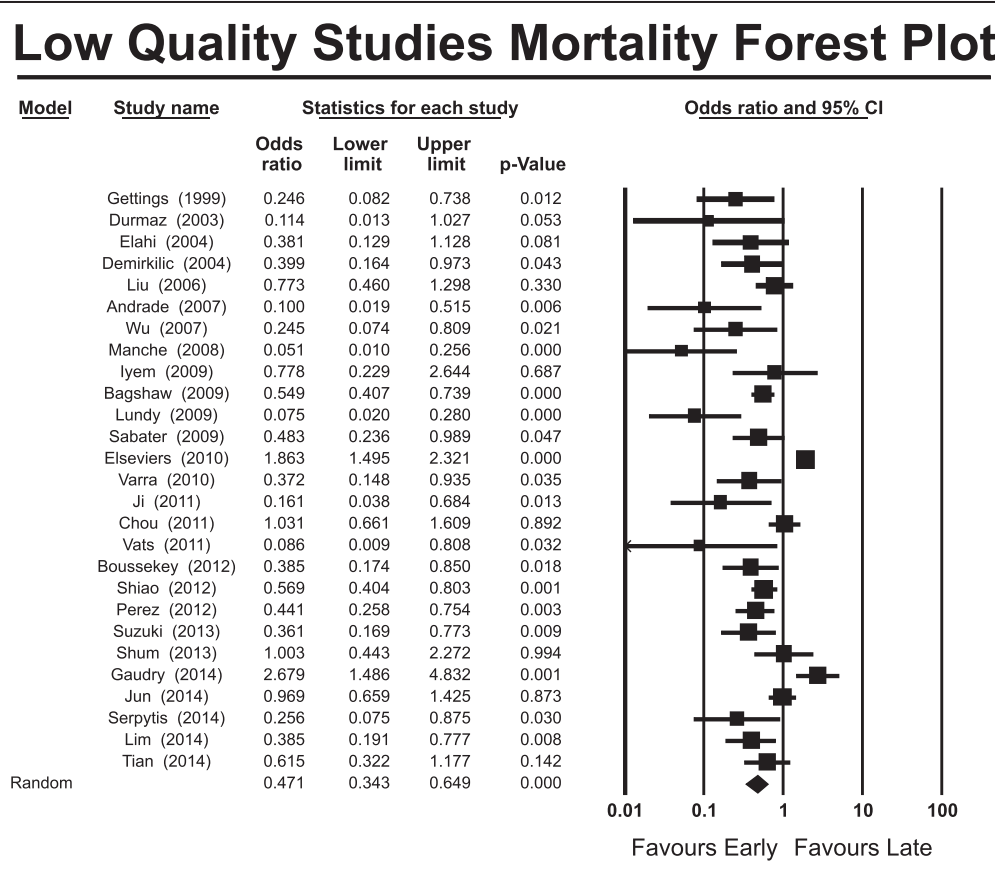

$\mathbf{n}=\mathbf{2 7}$

Fig. 2 Mortality forest plot pooled analysis of low-quality studies $(n=27)$ 


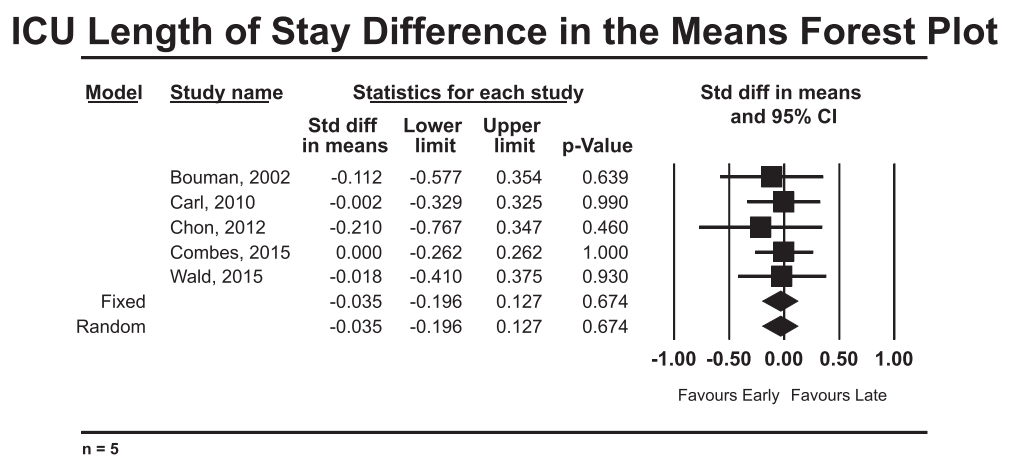

Fig. 3 Forest plot of pooled analysis of standard difference of the means for intensive care unit length of stay $(n=5)$

model with a standard difference in the means of 0.040 (95\% CI -0.125 to $0.204, p=0.638$ ) (Fig. 4).

\section{Discussion}

Despite several studies having been conducted on this topic over the last 30 years, a clear answer regarding the optimal timing of RRT in critical illness remains elusive. Our analysis does not confirm the conclusions of previous meta-analyses on this topic. Four studies [12, 14, 21, 35] in the high-quality group were previously included in the meta-analysis by Karvellas et al. [9], and only one study [12] was included in the meta-analysis by Seabra et al. [8]. The addition of four recently published studies $[15-17,40]$ and one high-quality study that was not previously included in meta-analysis [7] accounts for our results that differ from those of earlier authors. Our conclusions build on the concerns raised by both earlier meta-analyses that the results of cohort trials in favor of "early" RRT were not reproduced in methodologically more rigorous study designs (i.e., RCTs). In our further analysis we did not identify critical illness patient subgroups for whom "early" RRT might be more beneficial. Similarly, how one defines early (according to time or on the basis of biochemical characteristics) does not identify a survival advantage associated with "early" RRT compared with usual care. The optimal timing for initiation of RRT is not clarified on the basis of research evaluated to date.

The strength of our present analysis rests on our extensive literature search and strict classification according to study quality to limit risk of type I hypothesis testing error. Prior meta-analyses relied heavily on retrospective cohort study data that possessed incomplete preintervention data or preexisting significant differences in groups which predisposed the investigators to identify a survival difference attributed to "early" RRT that may have been accounted for by the preintervention population differences. We identified differences in the crude mortality rates between the high- and low-quality studies that are incompletely explained. The crude mortality rate differences may be explained by factors that are not adequately controlled for between the groups before the intervention of "early" versus "late" RRT (e.g., unreported regional institutional differences, variation in intensive care resources, institutional setting variability [academic versus community], or natural history variability of the diseases precipitating critical illness). In cohort trials, a difference in preintervention study groups indicates a

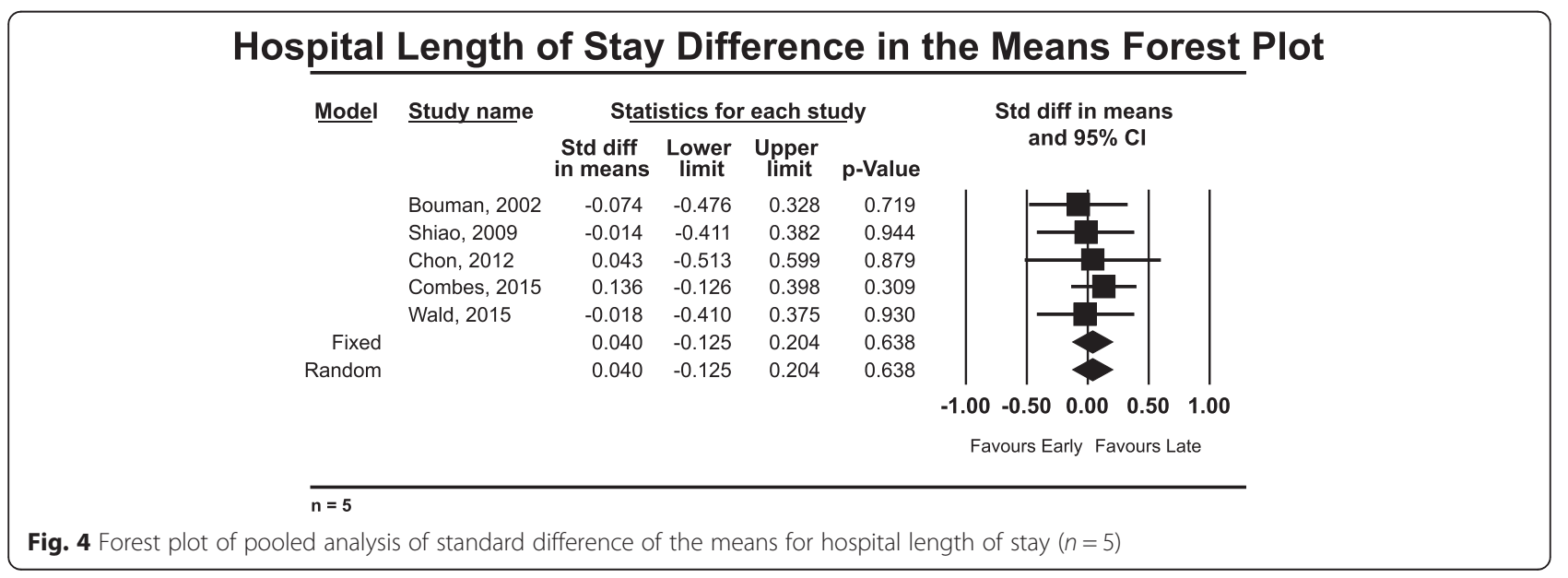


critical methodological flaw that precludes deriving conclusions from their results. This is referred to as a type I error in hypothesis testing and may falsely attribute differences in outcomes to the study variable rather than the differences between cohorts that existed before analysis. Among high-quality studies, there was no survival advantage to "early" RRT with an OR of $0.665(p=0.146)$. Any inclusion of the low-quality study data would significantly pull the conclusion in favor of "early" RRT, which would represent fulfillment of a type I statistical hypothesis error. The strength of our work is that we vigorously guarded against this possibility.

Subgroup analysis of the high-quality studies did not reveal a survival benefit associated with either a surgical or medical critical care patient population. This conclusion remained the same regardless of whether early was defined by time or on the basis of biochemical parameters. Our secondary outcome analysis was limited by inconsistent and incomplete data reported across studies. Limited pooled analysis of the available data suggested that there was no significant effect on either ICU or hospital LOS associated with "early" RRT. Incomplete data does not permit us to evaluate additional secondary outcomes of interest (such as requirement for mechanical ventilation or rates of renal recovery) that might also be clinically relevant considerations factored into the decision to initiate RRT in critical illness.

By limiting our analysis to studies meeting high-quality criteria, we dismissed a large volume of research on this topic. A critique of our work is that we discarded studies for methodological shortcomings that others may feel should have been included. Most studies $(n=21)$ in the low-quality group were excluded for incomplete cohort data or significant preintervention differences between cohort groups. The decision to exclude these trials is less controversial than our decision to exclude cohort trials for an NOQA Scale rating less than $7(n=6)$. This is potentially controversial because the NOQA Scale has received criticism regarding its validity and applicability in metaanalysis cohort trial quality assessment [50]. The NOQA Scale has received positive endorsement from some authors [11], but detailed psychometric properties have not been published in peer-reviewed journals to date. Furthermore, our selection of an NOQA Scale rating less than 7 to identify low quality is arbitrary. Our rationale for selecting this cutoff was that it necessitates that at least one of the three NOQA Scale domains be seriously compromised, and we felt that this represented a significant bias predisposing the study results to committing a type I error pattern. Seabra et al. [8] attempted to assign a quality score to trials $(0=$ lowest quality to $5=$ best quality) to evaluate this domain, but their methodology for score assignment was obscure and was not able to be replicated or directly compared with our methods. In qualitative comparison, the study assigned their top score [12] was included in our quantitative analysis; however, their second highest quality study [13] was excluded due to lack of reported illness severity scores between groups. Including studies with methodological errors does not advance scientific understanding of this topic and has contributed to the discordant findings on it.

Early studies on this topic were small and may have overestimated an effect size associated with "early" RRT based on the small size of the study populations. An example of this problem is the Sugahara et al. study [14], where 14-day mortality within the "early" group was $14 \%$ (2 of 14), compared with $86 \%$ (12 of 14) in the "late" group $(p<0.01)$. While this study was included in our quantitative analysis, the magnitude of the mortality benefit reported in this trial associated with "early" RRT has not been reproduced by subsequent investigators, for reasons that are not clear. In our review of the ongoing trials on this topic registered with the NIH (www.clinical Trials.gov), we identified three trials [51-53] that may add to knowledge in this area. The methodology of all three active RCTs is roughly similar, with patients randomized from a point in time triggered by the development of biochemical renal injury reflected by a RIFLE grade of "failure" (at least one of rise in creatinine by minimum of $300 \%$, oliguria less than $0.3 \mathrm{ml} / \mathrm{kg} / \mathrm{h}$ for $12 \mathrm{~h}$, or anuria lasting more than $12 \mathrm{~h}$ ). From this biochemical entry point, patients will be randomized to immediate initiation of RRT (goal time to RRT less than $12 \mathrm{~h}$ ) or standard care (RRT initiated after failure of medical management to temporize metabolic derangements or volume overload). These study designs are similar to the design used by Wald et al. [17], included in our analysis, that was able to separate an "early" group to mean time to RRT of $9.2 \mathrm{~h}$ and a "late" RRT group with a mean time to RRT of $32 \mathrm{~h}$ after biochemical inclusion criteria were met. Wald et al. [17] did not identify a significant difference in mortality rates between their two groups $(p=0.74)$. These studies in process will add to the quantity of patients evaluated in this manner and will build on the availability of highquality data on this topic. By clearly defining routine biochemical criteria associated with acute renal injury, they provide a practical method of renal injury assessment that can be determined by intensivists and nephrologists considering RRT.

\section{Conclusions}

The results of our meta-analysis contradict the findings reported by previous authors $[8,9]$, and we conclude that "early" initiation of RRT in critically ill patients with AKI does not improve survival. This conclusion is derived from the pooled high-quality trial data and excludes data from cohort trials where there were methodological shortcomings that predisposed them to find an effect 
misattributed to the intervention. Pooled analysis of secondary outcomes did not demonstrate a statistical reduction in ICU or hospital LOS. Additional well-designed RCTs will provide greater confidence in these conclusions as optimal patient care practices progress in critical care. Clinical triggers for the initiation of RRT to optimize patient outcomes have not been clearly identified by current research. Meanwhile, intensivists and nephrologists are encouraged to refrain from lowering their clinical thresholds for implementing RRT in critical care patients with acute renal injury.

\section{Key messages}

- High-quality trial data do not demonstrate improved survival using an "early" RRT approach in critical illness complicated by AKI.

- Lower-quality trial data demonstrate significantly higher mortality rates and form the basis for the bulk of support for "early" AKI.

- The optimal time to initiate RRT in critical illness remains undefined.

- A conservative approach to initiating RRT in critical illness is supported.

\section{Additional files}

Additional file 1: Figure S1. Preferred Reporting Items for Systematic Reviews and Meta-Analyses (PRISMA) checklist. (PDF 51 kb)

Additional file 2: Table S1. Search terms used during literature review. (DOCX $15 \mathrm{~kb}$ )

Additional file 3: Figure S2. Article selection process. (PDF $86 \mathrm{~kb}$ )

Additional file 4: Figure S3. a Mortality forest plot of subgroup analysis of high-quality studies according to post-surgical ICU admission type $(n=3)$. $\mathbf{b}$ Mortality forest plot of subgroup analysis of high-quality studies according to medical ICU admission type $(n=6)$. (ZIP $120 \mathrm{~kb})$

Additional file 5: Figure S4. a Mortality forest plot of subgroup analysis of high-quality studies based on the definition of "early" according to time criteria (hours or days) $(n=4)$. b Mortality forest plot of subgroup analysis of high-quality studies based on the definition of "early" according to biochemical parameters (i.e., rising creatinine, uremia, oliguria) $(n=5)$. (ZIP $121 \mathrm{~kb})$

\section{Abbreviations}

AKI: acute kidney injury; AKIN: Acute Kidney Injury Network; AMA: against medical advice; APACHE2: Acute Physiology and Chronic Health Evaluation II; ARDS: acute respiratory distress syndrome; BUN: blood urea nitrogen; CKD: chronic kidney disease; Cr: creatinine; CRF: chronic renal failure; CRRT: continuous renal replacement therapy; EHV: early high volume hemofiltration; ELV: early low volume hemofiltration; eGFR: estimated glomerular filtration rate; ESRD: end-stage renal disease; ICU: intensive care unit; ISS: illness severity score; LLV: late low volume hemofiltration; LOS: length of stay; NIH: National Institutes of Health; NOQA: NewcastleOttawa Quality Assessment; NR: not reported; NS: not significant; OR: odds ratio; PRISMA: Preferred Reporting Items for Systematic Reviews and MetaAnalyses; RCT: randomized controlled trial; RIFLE: risk, injury, failure, loss of function, and end-stage kidney disease; RPGN: rapidely progressive glomerulonephritis; RR: relative risk; RRT: renal replacement therapy; SAPS2: Simplified Acute Physiology Score II; SHARF: Stuivenberg Hospital Acute Renal Failure score below; SOFA: Sequential Organ Failure Assessment;
SRIFLE: simple criteria for risk injury failure loss of function and end-stage kidney disease; UOP: urine output; NR: not reported.

\section{Competing interests}

The authors declare that they have no competing interests.

\section{Authors' contributions}

BTW performed the literature search, reviewed studies for inclusion, performed the pooled data analysis using CMA software, and wrote the manuscript. RLCK acted as chair for article review and inclusion, reviewed studies for inclusion, provided senior oversight during manuscript development, and was primary editor of the manuscript during revisions. SK provided oversight for the literature search with the NIH librarian, reviewed studies for inclusion, and contributed to manuscript review. $\mathrm{SA}, \mathrm{XB}$, and GWB reviewed studies for inclusion and contributed to manuscript review. All authors contributed to and also read and approved the final version of the manuscript submitted for publication.

\section{Author details}

${ }^{1}$ Division of Internal Medicine, Department of Medicine, University of Calgary, Calgary, AB, Canada. ${ }^{2}$ Harvard School of Public Health, Harvard University, Boston, MA, USA. ${ }^{3}$ Division of Critical Care Medicine, Department of Medicine, Western University, 800 Commissioner's Road East, London, ON N6A 5W9, Canada.

Received: 14 December 2015 Accepted: 8 April 2016

Published online: 06 May 2016

\section{References}

1. Ahlström A, Tallgren M, Peltonen S, Räsänen P, Pettilä V. Survival and quality of life of patients requiring acute renal replacement therapy. Intensive Care Med. 2005;31(9):1222-8.

2. Bagshaw SM, Laupland KB, Doig CJ, Mortis G, Fick GH, Mucenski M, et al. Prognosis for long-term survival and renal recovery in critically ill patients with severe acute renal failure: a population-based study. Crit Care. 2005; 9(6):R700-9.

3. Hoste EA, Clermont G, Kersten A, Venkataraman R, Angus DC, De Bacquer D et al. RIFLE criteria for acute kidney injury are associated with hospital mortality in critically ill patients: a cohort analysis. Crit Care. 2006;10(3):R73.

4. Uchino S, Kellum JA, Bellomo R, Doig GS, Morimatsu H, Morgera S, et al. Acute renal failure in critically ill patients: a multinational, multicenter study. JAMA. 2005;294(7):813-8.

5. Wald R, Quinn RR, Luo J, Li P, Scales DC, Mamdani MM, et al. Chronic dialysis and death among survivors of acute kidney injury requiring dialysis. JAMA. 2009;302(11):1179-85.

6. Salisbury PF. Timely versus delayed use of the artificial kidney. AMA Arch Intern Med. 1958;101(4):690-701.

7. Payen D, Mateo J, Cavaillon JM, Fraisse F, Floriot C, Vicaut E, et al. Impact of continuous venovenous hemofiltration on organ failure during the early phase of severe sepsis: a randomized controlled trial. Crit Care Med. 2009; 37(3):803-10.

8. Seabra VF, Balk EM, Liangos O, Sosa MA, Cendoroglo M, Jaber BL. Timing of renal replacement therapy initiation in acute renal failure: a meta-analysis. Am J Kidney Dis. 2008;52(2):272-84

9. Karvellas C, Farhat M, Sajjad I, Mogensen S, Bagshaw S. Timing of initiation of renal replacement therapy in acute kidney injury: a meta-analysis [abstract]. Crit Care Med. 2010;38(12 Suppl):A105

10. Liberati A, Altman DG, Tetzlaff J, Mulrow C, Gøtzsche PC, loannidis JP, et al. The PRISMA statement for reporting systematic reviews and meta-analyses of studies that evaluate healthcare interventions: explanation and elaboration. BMJ. 2009;339:b2700.

11. Deeks JJ, Dinnes J, D'Amico R, Sowden AJ, Sakarovitch C, Song F, et al. Evaluating non-randomised intervention studies. Health Technol Assess. 2003;7(27):iii-x, 1-173.

12. Bouman CS, Oudemans-Van Straaten HM, Tijssen JG, Zandstra DF, Kesecioglu J. Effects of early high-volume continuous venovenous hemofiltration on survival and recovery of renal function in intensive care patients with acute renal failure: a prospective, randomized trial. Crit Care Med. 2002;30(10):2205-11. 
13. Durmaz I, Yagdi T, Calkavur T, Mahmudov R, Apaydin AZ, Posacioglu H, et al. Prophylactic dialysis in patients with renal dysfunction undergoing on-pump coronary artery bypass surgery. Ann Thorac Surg. 2003;75(3):859-64.

14. Sugahara S, Suzuki H. Early start on continuous hemodialysis therapy improves survival rate in patients with acute renal failure following coronary bypass surgery. Hemodial Int. 2004;8(4):320-5.

15. Jamale TE, Hase NK, Kulkarni M, Pradeep KJ, Keskar V, Jawale S, et al. Earlier-start versus usual-start dialysis in patients with community-acquired acute kidney injury: a randomized controlled trial. Am J Kidney Dis. 2013;62(6):1116-21.

16. Combes A, Bréchot N, Amour J, Cozic N, Lebreton G, Guidon C, et al. Early high-volume hemofiltration versus standard care for post-cardiac surgery shock: the HEROICS Study. Am J Respir Crit Care Med. 2015;192(10):1179-90.

17. Wald R, Adhikari NK, Smith OM, Weir MA, Pope K, Cohen A, et al. Comparison of standard and accelerated initiation of renal replacement therapy in acute kidney injury. Kidney Int. 2015;88(4):897-904.

18. Liu KD, Himmelfarb J, Paganini E, Ikizler TA, Soroko SH, Mehta RL, et al. Timing of initiation of dialysis in critically ill patients with acute kidney injury. Clin J Am Soc Nephrol. 2006;1(5):915-9.

19. Iyem H, Tavli M, Akcicek F, Buket S. Importance of early dialysis for acute renal failure after an open-heart surgery. Hemodial Int. 2009;13(1):55-61.

20. Bagshaw SM, Uchino S, Bellomo R, Morimatsu H, Morgera S, Schetz M, et al. Timing of renal replacement therapy and clinical outcomes in critically ill patients with severe acute kidney injury. J Crit Care. 2009;24(1):129-40.

21. Shiao CC, Wu VC, Li WY, Lin YF, Hu FC, Young GH, et al. Late initiation of renal replacement therapy is associated with worse outcomes in acute kidney injury after major abdominal surgery. Crit Care. 2009;13(5):R171.

22. Sabater J, Perez XL, Albertos R, Gutierrez D, Labad X. Acute renal failure in septic shock: Should we consider different continuous renal replacement therapies on each RIFLE score stage? [abstract 0924]. Intensive Care Med. 2009;35 Suppl 1:S239.

23. Elseviers MM, Lins RL, Van der Niepen P, Hoste E, Malbrain ML, Damas $P$, et al. Renal replacement therapy is an independent risk factor for mortality in critically ill patients with acute kidney injury. Crit Care. 2010;14(6):R221.

24. Vaara ST, Korhonen AM, Kaukonen KM, Nisula S, Inkinen O, Hoppu S, et al, Early initiation of renal replacement therapy is associated with decreased risk for hospital mortality in the critically ill [abstract]. Intensive Care Med. 2012;38 Suppl 1:S9.

25. Perez X, Sabater J, Huguet M, Santafosta E, Lopez JC, Alonso V, et al. Early timing in septic shock patients [abstract]. Intensive Care Med. 2012;38 Suppl 1:S263.

26. Jun M, Bellomo R, Cass A, Gallagher M, Lo S, Lee J. Timing of renal replacement therapy and patient outcomes in the randomized evaluation of normal versus augmented level of replacement therapy study. Crit Care Med. 2014;42(8):1756-65.

27. Lim CC, Tan CS, Kaushik M, Tan HK. Initiating acute dialysis at earlier Acute Kidney Injury Network stage in critically ill patients without traditional indications does not improve outcome: a prospective cohort study. Nephrology (Carlton). 2015;20(3):148-54.

28. Gettings LG, Reynolds HN, Scalea T. Outcome in post-traumatic acute renal failure when continuous renal replacement therapy is applied early vs. late. Intensive Care Med. 1999;25(8):805-13.

29. Elahi MM, Lim MY, Joseph RN, Dhannapuneni RR, Spyt TJ. Early hemofiltration improves survival in post-cardiotomy patients with acute renal failure. Eur J Cardiothorac Surg. 2004;26(5):1027-31.

30. Demirkiliç U, Kuralay E, Yenicesu M, Cağlar K, Oz BS, Cingöz F, et al. Timing of replacement therapy for acute renal failure after cardiac surgery. J Card Surg. 2004;19(1):17-20

31. Andrade L, Cleto S, Seguro AC. Door-to-dialysis time and daily hemodialysis in patients with leptospirosis: impact on mortality. Clin J Am Soc Nephrol. 2007;2(4):739-44.

32. Wu VC, Ko WJ, Chang HW, Chen YS, Chen YW, Chen YM, et al. Early renal replacement therapy in patients with postoperative acute liver failure associated with acute renal failure: effect on postoperative outcomes. J Am Coll Surg. 2007;205(2):266-76.

33. Manche A, Casha A, Rychter J, Farrugia E, Debono M. Early dialysis in acute kidney injury after cardiac surgery. Interact Cardiovasc Thorac Surg. 2008; 7(5):829-32.

34. Lundy JB, Chung KK, Wolf SF, Barillo DJ, King BT, White CE, et al. Improvement in mortality in severely burned patients with acute lung injury/acute respiratory distress syndrome and acute kidney injury with early continuous venovenous hemofiltration. Blood Purif. 2009;27(3):278-9.
35. Carl DE, Grossman C, Behnke M, Sessler CN, Gehr TW. Effect of timing of dialysis on mortality in critically ill, septic patients with acute renal failure. Hemodial Int. 2010;14(1):11-7.

36. Ji Q, Mei Y, Wang X, Feng J, Cai J, Zhou Y, et al. Timing of continuous veno-venous hemodialysis in the treatment of acute renal failure following cardiac surgery. Heart Vessels. 2011;26(2):183-9.

37. Chou YH, Huang TM, Wu VC, Wang CY, Shiao CC, Lai CF, et al. Impact of timing of renal replacement therapy initiation on outcome of septic acute kidney injury. Crit Care. 2011;15(3):R134.

38. Vats HS, Dart RA, Okon TR, Liang H, Paganini EP. Does early initiation of continuous renal replacement therapy affect outcome: experience in a tertiary care center. Ren Fail. 2011;33(7):698-706.

39. Boussekey N, Capron B, Delannoy PY, Devos P, Alfandari S, Chiche A, et al Survival in critically ill patients with acute kidney injury treated with early hemodiafiltration. Int J Artif Organs. 2012;35(12):1039-46.

40. Chon GR, Chang JW, Huh JW, Lim CM, Koh Y, Park SK, et al. A comparison of the time from sepsis to inception of continuous renal replacement therapy versus RIFLE criteria in patients with septic acute kidney injury. Shock. 2012;38(1):30-6

41. Shiao CC, Ko WJ, Wu VC, Huang TM, Lai CF, Lin YF, et al. U-curve association between timing of renal replacement therapy initiation and in-hospital mortality in postoperative acute kidney injury. PLoS One. 2012;7(8):e42952.

42. Suzuki J, Ohnuma T, Sanayama H, Ito K, Fujiwara T, Yamada H, et al. Early initiation of continuous renal replacement therapy is associated with lower mortality in patients with acute kidney injury [abstract]. Intensive Care Med. 2013;39 Suppl 2:S443.

43. Shum HP, Chan KC, Kwan MC, Yeung AW, Cheung EW, Yan WW. Timing for initiation of continuous renal replacement therapy in patients with septic shock and acute kidney injury. Ther Apher Dial. 2013;17(3):305-10.

44. Gaudry S, Ricard JD, Leclaire C, Rafat C, Messika J, Bedet A, et al. Acute kidney injury in critical care: experience of a conservative strategy. J Crit Care. 2014;29(6):1022-7.

45. Serpytis P, Smigelskaite A, Katliorius R, Griskevicius A. The early use of hemofiltration increases survival in patients with acute renal failure in the setting of intensive cardiac care unit [abstract PM046]. Glob Heart. 2014;9(1 Suppl):e70.

46. Tian H, Sun T, Hao D, Wang T, Li Z, Han S, et al. The optimal timing of continuous renal replacement therapy for patients with sepsis-induced acute kidney injury. Int Urol Nephrol. 2014;46(10):2009-14.

47. Zygun DA, Laupland KB, Fick GH, Sandham JD, Doig CJ. Limited ability of SOFA and MOD scores to discriminate outcome: a prospective evaluation in 1,436 patients. Can J Anaesth. 2005;52(3):302-8.

48. Pettilä V, Pettilä M, Sarna S, Voutilainen P, Takkunen O. Comparison of multiple organ dysfunction scores in the prediction of hospital mortality in the critically ill. Crit Care Med. 2002;30(8):1705-11.

49. Vincent JL, Moreno R. Clinical review: scoring systems in the critically ill. Crit Care. 2010;14(2):207.

50. Stang A. Critical evaluation of the Newcastle-Ottawa scale for the assessment of the quality of nonrandomized studies in meta-analyses. Eur J Epidemiol. 2010;25(9):603-5.

51. Gaudry S, Hajage D, Schortgen F, Martin-Lefevre L, Tubach F, Pons B, et al. Comparison of two strategies for initiating renal replacement therapy in the intensive care unit: study protocol for a randomized controlled trial (AKIKI). Trials. 2015;16:170.

52. Barbar SD, Binquet $C$, Monchi $M$, Bruyère $R$, Quenot JP. Impact on mortality of the timing of renal replacement therapy in patients with severe acute kidney injury in septic shock: the IDEAL-ICU study (Initiation of Dialysis EArly Versus deLayed in Intensive Care Unit): study protocol for a randomized controlled trial. Trials. 2014:15:270.

53. Smith OM, Wald R, Adhikari NK, Pope K, Weir MA, Bagshaw SM, et al. Standard versus accelerated initiation of renal replacement therapy in acute kidney injury (STARRT-AKI): study protocol for a randomized controlled trial. Trials. 2013:14:320. 